\title{
Boundary Regularity for the Porous Medium Equation
}

\author{
ANDERS BJÖRN@D, JANA BJÖRN@D, Ugo GIANAZZA@ \& \\ JUHANA SilJANDER
}

Communicated by P. -L. Lions

\begin{abstract}
We study the boundary regularity of solutions to the porous medium equation $u_{t}=\Delta u^{m}$ in the degenerate range $m>1$. In particular, we show that in cylinders the Dirichlet problem with positive continuous boundary data on the parabolic boundary has a solution which attains the boundary values, provided that the spatial domain satisfies the elliptic Wiener criterion. This condition is known to be optimal, and it is a consequence of our main theorem which establishes a barrier characterization of regular boundary points for general—not necessarily cylindrical—domains in $\mathbf{R}^{n+1}$. One of our fundamental tools is a new strict comparison principle between sub- and superparabolic functions, which makes it essential for us to study both nonstrict and strict Perron solutions to be able to develop a fruitful boundary regularity theory. Several other comparison principles and pasting lemmas are also obtained. In the process we obtain a rather complete picture of the relation between sub/superparabolic functions and weak sub/supersolutions.
\end{abstract}

\section{Introduction}

Let $\Theta$ be a bounded open set in a Euclidean space and for every $f \in C(\partial \Theta)$ let $u_{f}$ be the solution of the Dirichlet problem with boundary data $f$ for a given partial differential equation. Then a boundary point $\xi_{0} \in \partial \Theta$ is regular if

$$
\lim _{\Theta \ni \zeta \rightarrow \xi_{0}} u_{f}(\zeta)=f\left(\xi_{0}\right) \text { for all } f \in C(\partial \Theta),
$$

i.e. if the solution to the Dirichlet problem attains the given boundary data continuously at $\xi_{0}$, for all continuous boundary data $f$.

In this paper, we characterize regular boundary points for the porous medium equation

$$
\partial_{t} u=\Delta u^{m}
$$


in terms of families of barriers, in the so-called degenerate case $1<m<\infty$, and for general (not necessarily cylindrical) domains. To our knowledge, Abdulla [1,2] is the only one who has studied the Dirichlet problem for the porous medium equation in noncylindrical domains.

The characterization of regular boundary points for different partial differential equations has a very long history. Poincaré [40] was the first to use barriers, while Lebesgue [33] coined the name. At that time, barriers were used to study the solvability of the Dirichlet problem for harmonic functions, a question that was later completely settled using, e.g., Perron solutions. In 1924, Lebesgue [34] characterized regular boundary points for harmonic functions by the existence of barriers. The corresponding characterization for the heat equation was given by Bauer [8] in 1962 , but barriers had then already been used to study boundary regularity for the heat equation since Petrovskiı [39] in 1935; see the introduction in [11] for more on the history of boundary regularity for the heat equation.

Coming to nonlinear parabolic equations of degenerate and singular types, the potential theory for $p$-parabolic equations was initiated by Kilpeläinen and Lindqvist in [28]. They established the parabolic Perron method, and also suggested a boundary regularity characterization in terms of one barrier. Even if the single barrier criterion has turned out to be problematic, [28] has been the basis for the further development by Lindqvist [35], Björn-Björn-Gianazza [9] and BjörnBjörn-Gianazza-Parviainen [10] for the p-parabolic equation

$$
\partial_{t} u=\Delta_{p} u:=\operatorname{div}\left(|\nabla u|^{p-2} \nabla u\right) .
$$

For the porous medium equation (1.1), potential theory is largely at its inception, and so far not very much is known about the boundary behaviour of solutions in general domains. To our knowledge the main contributions in this field are due to Ziemer [45], Abdulla [1,2] and Kinnunen-Lindqvist-Lukkari [30].

Ziemer [45] studied boundary regularity in cylinders for a class of degenerate parabolic equations, which includes the porous medium equation with $m>1$, but with boundary data taken in a weak (Sobolev) sense; see Section 11 for further details.

Abdulla [1,2] investigated the Dirichlet problem for the porous medium equation with $m>0$ in general domains $\Theta \subset \mathbf{R}^{n+1}, n \geq 2$. Existence was established in [1], while uniqueness, comparison and stability theorems were presented in [2]. Therein, the smoothness condition on the boundary in order to have $u \in C(\bar{\Theta})$ is given in terms of a parabolic Hölder-type modulus; cf. Theorems 2.4 and 2.5 for the cylindrical case.

Kinnunen-Lindqvist-Lukkari [30] developed the Perron method for the porous medium equation in the degenerate range $m>1$ and showed that nonnegative continuous boundary functions are resolutive in arbitrary cylindrical domains. A boundary function $f$ is resolutive if the upper and lower Perron solutions $\bar{P} f$ and $\underline{P} f$ coincide.

The present paper can be considered as an extension of the previous contributions in several different but strictly related directions, as well as an initial development of a boundary regularity theory for the porous medium equation in terms of barriers. Under this second point of view, it is strictly related 
to the works [9] and [10] for the $p$-parabolic equation (1.2), even though the porous medium equation has extra difficulties not present for the $p$-parabolic equation. In particular, if $u$ is a solution of the porous medium equation (1.1) and $c \neq 0$ is a constant, then typically $u+c$ is not a solution. Moreover, we restrict ourselves to nonnegative functions, and therefore are not allowed to change sign.

It is possible to study sign-changing solutions of the porous medium equation, as has been done by some authors, but in addition to causing extra difficulties it may also cause significant differences when it comes to boundary regularity, as it seems quite possible that boundary regularity can be different for nonnegative and sign-changing functions. Here we restrict ourselves to nonnegative, and primarily positive, functions.

A well-known problem for the porous medium equation is the difficulty of obtaining a comparison principle between sub- and superparabolic functions. One of the main achievements in [30] was their comparison principle for cylinders (cf. Theorem 3.6). In order to even start developing the theory in this paper, it is fundamental to have a comparison principle in general domains, which we obtain in Theorem 5.1.

Comparison principles usually require an inequality $\leq$ on the boundary, and to establish such a comparison principle for general domains has been a major problem both for earlier authors and for us. We have chosen a slightly different and novel route obtaining a strict comparison principle in general domains, with the strict inequality $<$ at the boundary (see Theorems 5.1 and 5.3). Using a strict comparison principle causes extra complications, but we have still been able to develop a fruitful Perron and boundary regularity theory in general domains.

For thorough presentations of the theory of the porous medium equation, we refer the interested reader to Daskalopoulos-Kenig [19] and Vázquez [41]; see also DiBenedetto-Gianazza-Vespri [23]. We primarily deal with the degenerate case $m \geq 1$, but whenever possible we have given statements for general $m>0$. The singular case $0<m<1$ will be the object of future research.

The paper is organized as follows. Section 2 is devoted to some preliminary material. In particular, we recall the different concepts of solutions and sub/supersolutions, as well as various existence, uniqueness and stability results that will be essential later on.

Section 3 deals with the notions of sub- and superparabolic functions. In Theorem 3.5, we show that if $u$ is a weak supersolution then its lsc-regularization $u_{*}$ is superparabolic. A corresponding result for weak subsolutions is also obtained. (As we are not allowed to change sign, the theory for weak subsolutions does not follow directly from the corresponding theory for weak supersolutions.) We conclude the section by presenting the parabolic comparison principle for cylinders due to Kinnunen-Lindqvist-Lukkari [30], with a new proof.

In Section 4 we consider further results on sub/superparabolic functions: in particular, under proper conditions, sub/superparabolic functions are weak sub/supersolutions. In this way, we establish a rather complete understanding of the relation between weak sub/supersolutions and sub/superparabolic functions. 
Section 5 is devoted to a series of different comparison principles, for sub- and superparabolic functions, both of elliptic and parabolic types, and both of strict and nonstrict types. Several pasting lemmas are also obtained.

In Section 6 we deal with the Perron method, and with boundary regularity. We introduce the notion of upper regular points, as well as of lower regular points for positive (resp. nonnegative) boundary data. From here on we restrict ourselves to bounded open sets $\Theta \subset \mathbf{R}^{n+1}$. Moreover, the boundary data are always assumed to be bounded.

Section 7 is devoted to the characterization of an upper regular point in terms of a two-parameter family of barriers, with some related properties, whereas Section 8 deals with the characterization of a lower regular point for positive boundary data, in terms of another two-parameter family of barriers. This reflects the fact that we can neither add constants nor change sign, which is the crucial difference compared with the $p$-parabolic equation (1.2), where a single one-parameter family of barriers is necessary and sufficient (see [9] and [10]). In this paper, we do not develop the general theory of lower regularity for nonnegative boundary data.

In Section 9 we show that the earliest points are always regular, while in Section 10 we prove that upper regularity, as well as lower regularity (for positive boundary data), are independent of the future.

Section 11 collects the most important contributions of the paper. First, we show in Theorem 11.1 that the boundary regularity (for positive boundary data) of a lateral boundary point $\left(x_{0}, t_{0}\right) \in \partial U \times\left[t_{1}, t_{2}\right]$, with respect to the cylinder $U \times\left(t_{1}, t_{2}\right)$, is determined by the elliptic regularity of $x_{0}$ with respect to the spatial set $U$. This result is optimal in the sense that every harmonic function $u$ induces a time-independent solution $u^{1 / m}$ of the porous medium equation, and the Wiener criterion is a necessary and sufficient condition for boundary regularity of harmonic functions. Then, in Theorem 11.2 we give a unique solvability result in suitable finite unions of cylinders, which generalizes previous unique solvability results due to Abdulla [1,2], as well as the resolutivity result by Kinnunen-LindqvistLukkari [30] for general cylinders.

Finally, "Appendix A" is devoted to the proof of Theorem 3.4; we thought it better to postpone it, in order not to spoil the flow of the main arguments in Section 3.

\section{Preliminaries}

Let $\Theta$ be an open set in $\mathbf{R}^{n+1}, n \geq 2$. We write points in $\mathbf{R}^{n+1}$ as $\xi=(x, t)$, where $x \in \mathbf{R}^{n}$ and $t \in \mathbf{R}$. For $m>0$, we consider the porous medium equation

$$
\partial_{t} u=\Delta u^{m}:=\operatorname{div}\left(\nabla u^{m}\right)
$$

where, from now on, the gradient $\nabla$ and the divergence div are taken with respect to $x$. In this paper we only consider nonnegative solutions $u$. This equation is degenerate if $m>1$ and singular if $0<m<1$. For $m=1$ it is the usual heat equation. Observe that if $u$ satisfies (2.1), and $a \in \mathbf{R}_{+}$, then (in general) $a u$ and $u+a$ do not satisfy (2.1). 
All our cylinders are bounded space-time cylinders, i.e. of the form $U_{t_{1}, t_{2}}:=$ $U \times\left(t_{1}, t_{2}\right) \Subset \mathbf{R}^{n+1}$, where $U \Subset \mathbf{R}^{n}$ is open. We say that $U_{t_{1}, t_{2}}$ is a $C^{k, \alpha}$-cylinder if $U$ is $C^{k, \alpha}$-smooth. The parabolic boundary of $U_{t_{1}, t_{2}}$ is

$$
\partial_{p} U_{t_{1}, t_{2}}=\left(\bar{U} \times\left\{t_{1}\right\}\right) \cup\left(\partial U \times\left(t_{1}, t_{2}\right]\right) .
$$

We define the parabolic boundary of a finite union of open cylinders $U_{t_{j}, s_{j}}^{j}$ as follows:

$$
\partial_{p}\left(\bigcup_{j=1}^{N} U_{t_{j}, s_{j}}^{j}\right)=\left(\bigcup_{j=1}^{N} \partial_{p} U_{t_{j}, s_{j}}^{j}\right) \backslash \bigcup_{j=1}^{N} U_{t_{j}, s_{j}}^{j} .
$$

Note that the parabolic boundary is by definition compact. Further,

$$
B(x, r)=\left\{z \in \mathbf{R}^{n}:|z-x|<r\right\}
$$

stands for the usual Euclidean ball in $\mathbf{R}^{n}$. We also let

$$
\begin{aligned}
& \Theta_{T}=\{(x, t) \in \Theta: t<T\}, \\
& \Theta_{-}=\{(x, t) \in \Theta: t<0\}, \\
& \Theta_{+}=\{(x, t) \in \Theta: t>0\} .
\end{aligned}
$$

Let $U$ be a bounded open set in $\mathbf{R}^{n}$. As usual, $W^{1,2}(U)$ denotes the space of real-valued functions $u$ such that $u \in L^{2}(U)$ and the distributional first partial derivatives $\partial u / \partial x_{i}, i=1,2, \ldots, n$, exist in $U$ and belong to $L^{2}(U)$. We use the norm

$$
\|u\|_{W^{1,2}(U)}=\left(\int_{U}|u|^{2} d x+\int_{U}|\nabla u|^{2} d x\right)^{1 / 2} .
$$

The Sobolev space $W_{0}^{1,2}(U)$ with zero boundary values is the closure of $C_{0}^{\infty}(U)$ with respect to the Sobolev norm.

By the parabolic Sobolev space $L^{2}\left(t_{1}, t_{2} ; W^{1,2}(U)\right)$, with $t_{1}<t_{2}$, we mean the space of measurable functions $u(x, t)$ such that the mapping $x \mapsto u(x, t)$ belongs to $W^{1,2}(U)$ for a.e. $t_{1}<t<t_{2}$ and the norm

$$
\left(\int_{t_{1}}^{t_{2}} \int_{U}\left(|u(x, t)|^{2}+|\nabla u(x, t)|^{2}\right) d x d t\right)^{1 / 2}
$$

is finite. The definition of the space $L^{2}\left(t_{1}, t_{2} ; W_{0}^{1,2}(U)\right)$ is similar. Analogously, by the space $C\left(t_{1}, t_{2} ; L^{2}(U)\right)$, with $t_{1}<t_{2}$, we mean the space of measurable functions $u(x, t)$, such that the mapping $t \mapsto u(\cdot, t) \in L^{2}(U)$ is continuous in the time interval $\left[t_{1}, t_{2}\right]$. We can now introduce the notion of weak solution.

Definition 2.1. A function $u: \Theta \rightarrow[0, \infty]$ is a weak solution of equation (2.1) if whenever $U_{t_{1}, t_{2}} \Subset \Theta$, we have $u \in C\left(t_{1}, t_{2} ; L^{m+1}(U)\right), u^{m} \in L^{2}\left(t_{1}, t_{2} ; W^{1,2}(U)\right)$ and $u$ satisfies the integral equality

$$
\int_{t_{1}}^{t_{2}} \int_{U} \nabla u^{m} \cdot \nabla \varphi d x d t-\int_{t_{1}}^{t_{2}} \int_{U} u \partial_{t} \varphi d x d t=0 \text { for all } \varphi \in C_{0}^{\infty}\left(U_{t_{1}, t_{2}}\right) .
$$


Continuous weak solutions are called parabolic functions.

A function $u: \Theta \rightarrow[0, \infty]$ is a weak supersolution (subsolution) if whenever $U_{t_{1}, t_{2}} \Subset \Theta$, we have $u^{m} \in L^{2}\left(t_{1}, t_{2} ; W^{1,2}(U)\right)$ and the left-hand side above is nonnegative (nonpositive) for all nonnegative $\varphi \in C_{0}^{\infty}\left(U_{t_{1}, t_{2}}\right)$.

One can also consider sign-changing (and nonpositive) weak (sub/super)solutions, defined analogously, see Kinnunen-Lindqvist [29] for details. The general sign-changing theory is however much less developed than the theory for nonnegative functions. Moreover, it seems likely that regularity for sign-changing solutions of the porous medium equation may be quite different from regularity when restricted to positive or nonnegative solutions, which we have chosen to work with here. For simplicity, we will often omit weak, when talking of weak (sub/super)solutions.

In this paper, the name parabolic (and later sub/superparabolic) refers precisely to the porous medium equation (2.1), which is just one of many parabolic equations considered in the literature. A more specific terminology could be "porousparabolic" but for simplicity and readability we refrain from this nonstandard term.

Remark 2.2. In Definition 2.1, when dealing with the range $m>1$, one could actually require less (see below) on $u$, namely

$$
u \in C\left(t_{1}, t_{2} ; L^{2}(U)\right) \text { and } u^{(m+1) / 2} \in L^{2}\left(t_{1}, t_{2} ; W^{1,2}(U)\right) .
$$

This has been done e.g. in DiBenedetto-Gianazza-Vespri [23]. Roughly speaking, our notion of solution corresponds to using $u^{m}$ as a test function in the weak formulation (2.2), whereas assuming (2.3) amounts to using $u$. Such a choice seems more natural in a number of applications, but it seemingly introduces the extra difficulty that two different notions of solutions are needed, according to whether $m \leq 1$ or $m \geq 1$. However, it has recently been proved by Bögelein-LehteläSturm [15, Theorem 1.2], that for $m \geq 1$ the two notions are equivalent.

Locally bounded solutions are locally Hölder continuous: this result is due to different authors. A full account is given in Daskalopoulos-Kenig [19], DiBenedettoGianazza-Vespri [23] and Vázquez [41]. For $m>\frac{(n-2)_{+}}{n+2}$ solutions are automatically locally bounded, whereas for $0<m \leq \frac{(n-2)_{+}}{n+2}$ explicit unbounded solutions are known, and in order to guarantee boundedness, an extra assumption on $u$ is needed (see the discussions in DiBenedetto [21, Chapter V] and DiBenedettoGianazza-Vespri [23, Appendix B]). Although it plays no role in the following, it is worth mentioning that nonnegative solutions satisfy proper forms of Harnack inequalities (see [23]).

Next we will present a series of auxiliary results, which will be used later in the paper.

Besides the notion of weak solutions given in Definition 2.1, we need to be able to uniquely solve the Dirichlet problem in smooth cylinders. Given measurable nonnegative functions $u_{0}$ on $U \Subset \mathbf{R}^{n}$ and $g$ on the lateral boundary $\Sigma_{t_{1}, t_{2}}=$ $\partial U \times\left(t_{1}, t_{2}\right]$, we are interested in finding a weak solution $u=u(x, t)$ defined in $U_{t_{1}, t_{2}}$ that solves the boundary value problem 


$$
\begin{cases}\partial_{t} u=\Delta u^{m} & \text { in } U_{t_{1}, t_{2}}, \\ u\left(\cdot, t_{1}\right)=u_{0} & \text { in } U, \\ u^{m}=g & \text { in } \Sigma_{t_{1}, t_{2}} .\end{cases}
$$

We need to define in which sense the initial condition and the lateral boundary data are taken.

It is well known that for sufficiently smooth $U$, functions $f \in W^{1,2}(U)$ have boundary values $T_{\partial U} f$, called traces, on the boundary $\partial U$ (see e.g. DiBenedetto [22, Theorem 18.1]). Moreover, the linear trace map $T_{\partial U}$ maps $W^{1,2}(U)$ onto the space $W^{1 / 2,2}(\partial U) \subset L^{2}(\partial U)$, and $T_{\partial U} f=\left.f\right|_{\partial U}$ if $f \in W^{1,2}(U) \cap C(\bar{U})$. In the time dependent context, the trace operator can be naturally extended into a continuous linear map

$$
T_{\Sigma_{t_{1}, t_{2}}}: L^{2}\left(t_{1}, t_{2} ; W^{1,2}(U)\right) \longrightarrow L^{2}\left(t_{1}, t_{2} ; W^{1 / 2,2}(\partial U)\right) \subset L^{2}\left(\Sigma_{t_{1}, t_{2}}\right) .
$$

In Vázquez [41, Theorems 5.13 and 5.14] the following result is proved, which addresses the problem of existence and uniqueness in the framework of $L^{p}$ spaces. A somewhat analogous result is proved in Alt-Luckhaus [3].

Theorem 2.3. Let $m>0$ and let $U_{t_{1}, t_{2}}$ be a $C^{2, \alpha}$-cylinder. Also let $u_{0} \in L^{m+1}(U)$ be nonnegative and assume that there exists $\bar{g} \in L^{2}\left(t_{1}, t_{2} ; W^{1,2}(U)\right)$ such that

$$
T_{\Sigma_{t_{1}, t_{2}}}(\bar{g})=g
$$

and $\bar{g}, \partial_{t} \bar{g} \in L^{\infty}\left(U_{t_{1}, t_{2}}\right)$. Then there exists a unique weak solution $u$ in $U_{t_{1}, t_{2}}$ such that

(i) $T_{\Sigma_{t_{1}, t_{2}}}\left(u^{m}\right)=g$,

(ii) $u(\cdot, t) \rightarrow u_{0}$ in the $L^{1}(U)$ topology, as $t \rightarrow t_{1}$.

Finally, the comparison principle applies to these solutions: if $u$ and $\hat{u}$ are weak solutions corresponding to $g, u_{0}$ and $\hat{g}, \hat{u}_{0}$, respectively, with $u_{0} \leq \hat{u}_{0}$ a.e. in $U$ and $g \leq \hat{g}$ a.e. in $\Sigma_{t_{1}, t_{2}}$, then $u \leq \hat{u}$ a.e. in $U_{t_{1}, t_{2}}$.

We also need to consider the existence of continuous solutions. Under this point of view, if we assign continuous data on the whole parabolic boundary, we have the following result:

Theorem 2.4. Let $m>0$ and let $U_{t_{1}, t_{2}}$ be a $C^{1, \beta}$-cylinder, where $\beta=\frac{m-1}{m+1}$ if $m>1$ and $\beta>0$ if $0<m \leq 1$. Also let $h \in C\left(\partial_{p} U_{t_{1}, t_{2}}\right)$ be nonnegative. Then there is a unique function $u \in C\left(\bar{U}_{t_{1}, t_{2}}\right)$ that is parabolic in $U_{t_{1}, t_{2}}$ and takes the boundary values $u=h$ on the parabolic boundary $\partial_{p} U_{t_{1}, t_{2}}$.

Moreover, if $h \leq h^{\prime} \in C\left(\partial_{p} U_{t_{1}, t_{2}}\right)$ and $u^{\prime} \in C\left(\bar{U}_{t_{1}, t_{2}}\right)$ is the unique function corresponding to $h^{\prime}$ as above, then $u \leq u^{\prime}$ in $U_{t_{1}, t_{2}}$.

Variations of this second boundary value problem have been widely studied. Aronson-Peletier [6] and Gilding-Peletier [26] proved the unique existence as here, provided $U_{t_{1}, t_{2}}$ is a $C^{2, \alpha}$-cylinder, $m>1$, and one has homogeneous conditions $h=0$ on the lateral boundary. We need this unique existence for general boundary conditions, in which case the result can be seen as a consequence of Abdulla [1,2], DiBenedetto [20] and Vespri [42]; see the comments in the proof below. 
Proof. In [1,2], Abdulla studies the unique solvability of the Dirichlet problem with continuous boundary data $h$ in a general (not necessarily cylindrical) open set $\Theta \subset \mathbf{R}^{n+1}$. In particular, conditions $\mathscr{A}$ and $\mathscr{B}$ of [1, Theorem 2.1] ensure existence, whereas condition $\mathscr{M}$ of [2, Theorem 2.2] ensures uniqueness. When $\Theta$ is a cylinder, $\mathscr{A}$ and $\mathscr{B}$ coincide. It is not hard to verify that if $U$ is a bounded open $C^{1, \beta}$-smooth set, with $\beta$ as above, then at every point of the parabolic boundary $\partial_{p} U_{t_{1}, t_{2}}$ conditions $\mathscr{B}$ and $\mathscr{M}$ are satisfied, yielding the unique existence of a suitable solution in $C\left(\bar{U}_{t_{1}, t_{2}}\right)$.

As a matter of fact, Abdulla uses a definition of solution which is weaker than Definition 2.1. However, the existence of a function $u \in C\left(\bar{U}_{t_{1}, t_{2}}\right)$, that is parabolic (in our sense) in $U_{t_{1}, t_{2}}$ and takes the boundary values $u=h$ on the parabolic boundary $\partial_{p} U_{t_{1}, t_{2}}$, follows from DiBenedetto [20, Remark 1.2] (for $m>$ 1) and Vespri [42, Theorem 1.1 and Remarks (a) and (d)] (for $0<m \leq 1$ ). Using integration by parts it can be shown that this parabolic function is a solution in the sense of Abdulla.

Since solutions in the sense of Abdulla are unique, it follows that the parabolic function provided by [20] or [42] is the unique continuous weak solution of the boundary value problem.

Finally, the inequality $u \leq u^{\prime}$ follows from [2, Theorem 2.3].

Having considered existence and uniqueness, we also need the following stability result from Abdulla [2, Corollary 2.3]:

Theorem 2.5. Let $m>0$ and let $U_{t_{1}, t_{2}}$ be a $C^{1, \beta}$-cylinder, where $\beta=\frac{m-1}{m+1}$ if $m>1$ and $\beta>0$ if $0<m \leq 1$. Also let $h_{j} \in C\left(\partial_{p} U_{t_{1}, t_{2}}\right)$ be nonnegative, and let $u_{j} \in C\left(\bar{U}_{t_{1}, t_{2}}\right)$ be the corresponding solutions given by Theorem $2.4, j=0,1, \ldots$ If $\sup _{\partial_{p} U_{t_{1}, t_{2}}}\left|h_{j}-h_{0}\right| \rightarrow 0$ as $j \rightarrow \infty$, then $u_{j}$ tends to $u_{0}$ locally uniformly in $U \times\left(t_{1}, t_{2}\right]$ as $j \rightarrow \infty$.

We proceed by stating a comparison principle for sub- and supersolutions in cylinders. It was first proved in $\mathbf{R}^{1+1}$ by Aronson-Crandall-Peletier [5], and in $\mathbf{R}^{n+1}$ by Dahlberg-Kenig [16-18]. A further and somewhat different statement of the comparison principle is given in Abdulla [2, Theorem 2.3]. For the proof of the following statement, we refer the reader to Daskalopoulos-Kenig [19, pp. 10-12] and Vázquez [41, pp. 132-134].

Proposition 2.6. (Comparison principle for sub- and supersolutions) Let $m>0$ and let $U_{t_{1}, t_{2}}$ be a $C^{2}$-cylinder. Suppose $u$ and $v$ are a super-and a subsolution in $U_{t_{1}, t_{2}}$, respectively, such that $u^{m}, v^{m} \in L^{2}\left(U_{t_{1}, t_{2}}\right)$. Assume, furthermore, that

$$
\begin{cases}\left(v^{m}-u^{m}\right)_{+}(\cdot, t) \in W_{0}^{1,2}(U) & \text { for a.e. } t \in\left(t_{1}, t_{2}\right), \\ (v-u)_{+}\left(x, t_{1}\right)=0 & \text { for a.e. } x \in U .\end{cases}
$$

Then $0 \leq v \leq u$ a.e. in $U_{t_{1}, t_{2}}$.

Proposition 2.6 is the first of many comparison principles in this paper. This is the only one between sub- and supersolutions, but we will have several different 
parabolic (Theorems 3.6, 5.1 and 5.4) and one elliptic-type (Theorem 5.3) comparison principles for sub- and superparabolic functions. In addition, sub- and superparabolic functions will be defined using yet another type of comparison principle, for which we also have alternative versions in Proposition 3.8 and Remark 3.9.

\section{Definition of Superparabolic Functions}

Definition 3.1. A function $u: \Theta \subset \mathbf{R}^{n+1} \rightarrow[0, \infty]$ is superparabolic if

(i) $u$ is lower semicontinuous;

(ii) $u$ is finite in a dense subset of $\Theta$;

(iii) $u$ satisfies the following comparison principle on each $C^{2, \alpha}$-cylinder $U_{t_{1}, t_{2}} \Subset$ $\Theta$ : If $h \in C\left(\bar{U}_{t_{1}, t_{2}}\right)$ is parabolic in $U_{t_{1}, t_{2}}$ and $h \leq u$ on $\partial_{p} U_{t_{1}, t_{2}}$, then $h \leq u$ in $U_{t_{1}, t_{2}}$.

That $v$ is subparabolic is defined analogously, except that $v: \Theta \rightarrow[0, \infty)$ is upper semicontinuous and the inequalities are reversed, i.e. we require that if $h \geq v$ on $\partial_{p} U_{t_{1}, t_{2}}$, then $h \geq v$ in $U_{t_{1}, t_{2}}$.

Note that as with sub- and supersolutions we implicitly assume that sub- and superparabolic functions are nonnegative in this paper.

In Kinnunen-Lindqvist [29], Kinnunen-Lindqvist-Lukkari [30] and AvelinLukkari [7] they require (iii) in Definition 3.1 to hold for arbitrary compactly contained cylinders $U_{t_{1}, t_{2}} \Subset \Theta$. (In $[29,30]$ they use the name "viscosity supersolution" instead of superparabolic, while in [7] they call them "semicontinuous supersolutions".)

One of our first aims is to show that our Definition 3.1 is equivalent to the definition in $[7,29,30]$, when $m \geq 1$. This will take some effort and will only be completed at the end of this section. The reason for our unorthodox definition is that we want to establish Theorem 3.5, which we have not been able to prove without using our definition. Once Theorem 3.5 has been deduced we are able to show that our definition of sub- and superparabolic functions is equivalent to the one in [7,29,30], when $m \geq 1$, see Remark 3.9.

The following consequences of the definition of sub- and superparabolicity are almost immediate, so we leave the proof to the reader:

Lemma 3.2. The following hold for all $m>0$ :

(a) if $u$ and $v$ are superparabolic, then $\min \{u, v\}$ is superparabolic;

(b) if $u$ is finite in a dense set, then $u$ is superparabolic if and only if $\min \{u, k\}$ is superparabolic for $k=1,2, \ldots$;

(c) if $u$ and $v$ are subparabolic, then $\max \{u, v\}$ is subparabolic;

(d) if $v$ is subparabolic, then $v$ is locally bounded.

For a function $u$ we define the lsc-regularization of $u$ as

$$
u_{*}\left(\xi_{0}\right)=\underset{\xi \rightarrow \xi_{0}}{\operatorname{ess} \liminf _{i}} u(\xi)
$$

We also say that $u$ is $l s c$-regularized if $u_{*}=u$. Avelin-Lukkari [7] proved the following result: 
Theorem 3.3. Let $m \geq 1$ and let $u$ be a supersolution. Then,

$$
u_{*}(x, t)=u(x, t)
$$

at all Lebesgue points of $u$ such that $u(x, t)<\infty$. In particular, $u_{*}=u$ a.e., and $u_{*}$ is a lower semicontinuous representative of $u$.

Strictly speaking, Avelin-Lukkari [7] only considers $m>1$; the remaining case $m=1$ can be recovered from Kuusi [32] assuming $p=2$.

Similarly, for a function $u$ we define the usc-regularization of $u$ as

$$
u^{*}\left(\xi_{0}\right)=\underset{\xi \rightarrow \xi_{0}}{\operatorname{ess} \lim _{0} \sup u(\xi)}
$$

and say that $u$ is $u s c$-regularized if $u^{*}=u$.

Theorem 3.4. Let $m \geq 1$ and let $u$ be a subsolution. Then,

$$
u^{*}(x, t)=u(x, t)
$$

at all Lebesgue points of $u$. In particular, $u^{*}=u$ a.e., and $u^{*}$ is an upper semicontinuous representative of $u$.

Due to the structure of the porous medium equation, this is not a trivial consequence of Theorem 3.3, but needs to be proved separately. We postpone the proof of Theorem 3.4 to "Appendix A".

Note that we do not need to require that $u(x, t)$ is finite in Theorem 3.4, since $u$ is nonnegative and subsolutions are essentially bounded from above when $m \geq 1$; see Andreucci [4].

Theorem 3.5. Let $m \geq 1$. If $u$ is a supersolution then $u_{*}$ is superparabolic. Similarly, if $v$ is a subsolution then $v^{*}$ is subparabolic.

In a less precise form this result was stated just after Theorem 1.1 in AvelinLukkari [7], without proof. We therefore provide a complete proof of this result, and this is also the reason for our unorthodox definition of sub- and superparabolic functions. Once Remark 3.9 has been established below, it follows directly that Theorem 3.5 is also valid using the sub- and superparabolic definition used in $[7,29,30]$.

Proof. Assume first that $u$ is a supersolution. By Theorem 3.3, $u_{*}=u$ a.e., and thus also $u_{*}$ is a supersolution. We want to show that $u_{*}$ is superparabolic. Condition (i) follows from Theorem 3.3, while (ii) follows directly. For (iii), fix a $C^{2, \alpha}$-cylinder $U_{t_{1}, t_{2}} \Subset \Theta$ and let $h \in C\left(\bar{U}_{t_{1}, t_{2}}\right)$ be such that it is parabolic in $U_{t_{1}, t_{2}} \Subset \Theta$ and $h \leq u_{*}$ on $\partial_{p} U_{t_{1}, t_{2}}$.

According to Definition 2.1, this means that $h^{m} \in L^{2}\left(s_{1}, s_{2} ; W^{1,2}(V)\right)$ for every cylinder $V_{s_{1}, s_{2}} \Subset U_{t_{1}, t_{2}}$, but this is not enough to directly apply the comparison principle in Proposition 2.6, which would require $h^{m} \in L^{2}\left(t_{1}, t_{2} ; W^{1,2}(U)\right)$. We therefore proceed as follows. 
Let $\bar{h}_{j} \in C^{\infty}\left(\mathbf{R}^{n+1}\right)$ and $h_{j}=\left.\bar{h}_{j}\right|_{\partial_{p} U_{t_{1}, t_{2}}}$ be such that $0 \leq h_{j} \leq h$ on $\partial_{p} U_{t_{1}, t_{2}}$ and $\sup _{\partial_{p} U_{t_{1}, t_{2}}}\left|h_{j}-h\right| \rightarrow 0$, as $j \rightarrow \infty$. Using Theorem 2.3, we can extend $h_{j}$ so that it is a weak solution in $U_{t_{1}, t_{2}}$ which takes the boundary data $h_{j}$ in the sense of traces and which satisfies $h_{j}^{m}(\cdot, t) \in W^{1,2}(U)$ for a.e. $t \in\left(t_{1}, t_{2}\right)$. By the comparison principle in Proposition 2.6, $h_{j} \leq u_{*}$ a.e. in $U_{t_{1}, t_{2}}$. Since $h_{j}$ is continuous, and $u_{*}$ is lsc-regularized, it directly follows that $h_{j} \leq u_{*}$ everywhere in $U_{t_{1}, t_{2}}$.

Moreover, since the boundary data $h_{j}$ are continuous, DiBenedetto [20, Theorem, p. 421] implies that $h_{j} \in C\left(\bar{U}_{t_{1}, t_{2}}\right)$. Hence $h_{j}$ coincides with the solution provided by Theorem 2.4. Letting $j \rightarrow \infty$, we conclude from Theorem 2.5 that $h \leq u_{*}$ everywhere in $U_{t_{1}, t_{2}}$. Hence $u_{*}$ is superparabolic. The proof for subsolutions is analogous, using Theorem 3.4.

To establish the equivalence between our sub- and superparabolic functions and the ones used in $[7,29,30]$, we will also need the following parabolic comparison principle for sub- and superparabolic functions, which was obtained by KinnunenLindqvist-Lukkari [30, Theorem 3.3].

Theorem 3.6. (Parabolic comparison principle for cylinders) Let $m \geq 1$ and let $U_{t_{1}, t_{2}}$ be an arbitrary cylinder in $\mathbf{R}^{n+1}$. Suppose that $u$ is a bounded superparabolic function and $v$ is a bounded subparabolic function in $U_{t_{1}, t_{2}}$. Assume that

$$
\limsup _{U_{t_{1}, t_{2}} \ni(y, s) \rightarrow(x, t)} v(y, s) \leq \liminf _{U_{t_{1}, t_{2}} \ni(y, s) \rightarrow(x, t)} u(y, s)
$$

for all $(x, t) \in \partial_{p} U_{t_{1}, t_{2}}$. Then $v \leq u$ in $U_{t_{1}, t_{2}}$.

As the definition of superparabolic functions in [30] is slightly different from ours, some comments are in order. Since we also had difficulties understanding how they concluded that $u \leq v$ everywhere (and not just a.e.) at the end of their proof, we seize the opportunity to provide our own proof (based partly on the ideas in [30]).

Proof. Without loss of generality we can assume that both $u$ and $v$ are bounded. Using (3.1) and the compactness of $\partial_{p} U_{t_{1}, t_{2}}$, we can for each $\varepsilon_{j}=1 / j, j=$ $1,2, \ldots$, find $C^{2, \alpha}$-cylinders $U_{s_{j}, t_{2}}^{j}:=U^{j} \times\left(s_{j}, t_{2}\right) \Subset U \times\left(t_{1}, t_{2}\right]$ so that

$$
U^{1} \Subset U^{2} \Subset \cdots \Subset \bigcup_{j=1}^{\infty} U^{j}=U, \quad s_{1}>s_{2}>\cdots \rightarrow t_{1},
$$

and

$$
v^{m} \leq u^{m}+\varepsilon_{j}^{m} \text { in } U_{t_{1}, t_{2}} \backslash U_{s_{j}, t_{2}}^{j}, j=1,2, \ldots
$$

Since $u$ and $v$ are lower and upper semicontinuous, respectively, we can also find nonnegative $\bar{h}_{j} \in C^{\infty}\left(\mathbf{R}^{n}\right)$ such that

$$
v^{m} \leq \bar{h}_{j}^{m}+\varepsilon_{j}^{m} \leq u^{m}+\varepsilon_{j}^{m} \text { in } U_{t_{1}, t_{2}} \backslash U_{s_{j}, t_{2}}^{j}, j=1,2, \ldots
$$


As in the proof of Theorem 3.5, we use Theorem 2.3, together with DiBenedetto [20, Theorem, p. 421], to find weak solutions $h_{j}$ and $\hat{h}_{j}$ in $U_{s_{j}, t_{2}}^{j}$ which take the boundary data $\bar{h}_{j}$ and $\left(\bar{h}_{j}^{m}+\varepsilon_{j}^{m}\right)^{1 / m}$, respectively, both in the sense of traces and continuously on $\partial_{p} U_{s_{j}, t_{2}}^{j}$. The super/subparabolicity of $u$ and $v$ now yield

$$
u \geq h_{j} \quad \text { and } \quad v \leq \hat{h}_{j} \quad \text { in } U_{s_{j}, t_{2}}^{j}
$$

If we extend $h_{j}$ and $\hat{h}_{j}$ as $\bar{h}_{j}$ and $\left(\bar{h}_{j}^{m}+\varepsilon_{j}^{m}\right)^{1 / m}$ outside $U_{s_{j}, t_{2}}^{j}$, then also

$$
u \geq h_{j} \quad \text { and } \quad v \leq \hat{h}_{j} \quad \text { in } U_{t_{1}, t_{2}} \backslash U_{s_{j}, t_{2}}^{j} .
$$

Moreover,

$$
h_{j} \leq \hat{h}_{j} \leq h_{j}+\varepsilon_{j} \text { in } U_{t_{1}, t_{2}} \backslash U_{s_{j}, t_{2}}^{j} \text { and } h_{j} \leq \hat{h}_{j} \text { in } U_{s_{j}, t_{2}}^{j},
$$

by Proposition 2.6.

Now, Theorem 5.16.1 in DiBenedetto-Gianazza-Vespri [23] shows that both families $\left\{h_{j}\right\}_{j=1}^{\infty}$ and $\left\{\hat{h}_{j}\right\}_{j=1}^{\infty}$ are locally equicontinuous in $U_{t_{1}, t_{2}}$. Hence, Ascoli's theorem and a diagonal argument provide us with subsequences, also denoted $\left\{h_{j}\right\}_{j=1}^{\infty}$ and $\left\{\hat{h}_{j}\right\}_{j=1}^{\infty}$, which converge locally uniformly in $U_{t_{1}, t_{2}}$ to continuous functions $h$ and $\hat{h}$. Clearly, $h \leq \hat{h}$ and taking limits in (3.2) and (3.3) yields

$$
u \geq h \quad \text { and } \quad v \leq \hat{h} \quad \text { in } U_{t_{1}, t_{2}} \text {. }
$$

For each $j=1,2, \ldots$, Lemma 3.2 in Kinnunen-Lindqvist-Lukkari [30] implies that

$$
\int_{s_{j}}^{t_{2}} \int_{U^{j}}\left(\hat{h}_{j}-h_{j}\right)\left(\hat{h}_{j}^{m}-h_{j}^{m}\right) d x d t \leq C \varepsilon_{j},
$$

where $C$ depends on $U$ and the bounds for $u$ and $v$, but not on $j$. Taking into account (3.4), we thus conclude that

$$
0 \leq \int_{t_{1}}^{t_{2}} \int_{U}\left(\hat{h}_{j}-h_{j}\right)\left(\hat{h}_{j}^{m}-h_{j}^{m}\right) d x d t \leq C \varepsilon_{j} .
$$

Since $h_{j} \rightarrow h$ and $\hat{h}_{j} \rightarrow \hat{h}$ in $U_{t_{1}, t_{2}}$ and all the functions are uniformly bounded, dominated convergence implies that

$$
\int_{t_{1}}^{t_{2}} \int_{U}(\hat{h}-h)\left(\hat{h}^{m}-h^{m}\right) d x d t=0
$$

and hence $h=\hat{h}$ a.e. Finally, the continuity of $h$ and $\hat{h}$, together with (3.5), yields $v \leq \hat{h}=h \leq u$.

Remark 3.7. The above proof also shows that the function $h=\hat{h}$ is a weak solution in $U_{t_{1}, t_{2}}$. Indeed, the Caccioppoli inequality (Lemma 2.15 in KinnunenLindqvist [29]) shows that $\left|\nabla h_{j}^{m}\right|$ and $\left|\nabla \hat{h}_{j}^{m}\right|$ are uniformly bounded in $L^{2}(s, t$; $\left.W^{1,2}(V)\right)$ for every cylinder $V_{s, t} \Subset U_{t_{1}, t_{2}}$. Thus, there is a weakly converging subsequence, for which the integral identity (2.2) on $V_{s, t} \Subset U_{t_{1}, t_{2}}$ pertains. 
Proposition 3.8. Let $m \geq 1$. If $u$ is superparabolic in $\Theta$, then it satisfies the following comparison principle on each cylinder $U_{t_{1}, t_{2}} \Subset \Theta:$ If $h \in C\left(\bar{U}_{t_{1}, t_{2}}\right)$ is parabolic in $U_{t_{1}, t_{2}}$ and $h \leq u$ on $\partial_{p} U_{t_{1}, t_{2}}$, then $h \leq u$ in $U_{t_{1}, t_{2}}$.

Similarly, if $v$ is subparabolic in $\Theta, U_{t_{1}, t_{2}} \Subset \Theta$ is a cylinder, $h \in C\left(\bar{U}_{t_{1}, t_{2}}\right)$ is parabolic in $U_{t_{1}, t_{2}}$ and $h \geq v$ on $\partial_{p} U_{t_{1}, t_{2}}$, then $h \geq u$ in $U_{t_{1}, t_{2}}$.

Remark 3.9. This shows that our definition of sub- and superparabolic functions is equivalent to the one used in Kinnunen-Lindqvist [29], Kinnunen-LindqvistLukkari [30] and Avelin-Lukkari [7]. It also follows from Theorem 5.4 below, that one can equivalently assume that the comparison principle holds for all compactly contained finite unions of cylinders; this equivalence was also pointed out in [29, p. 147].

Whether it is equivalent to just assuming that the comparison principle holds for space-time boxes $\left(a_{1}, b_{1}\right) \times \ldots \times\left(a_{n}, b_{n}\right) \times\left(t_{1}, t_{2}\right)$ is an open problem. Such an equivalence is known to hold for the $p$-parabolic equation (1.2), see Korte-KuusiParviainen [31, Corollary 4.7].

Proof of Proposition 3.8. Let $u$ be superparabolic and let $U_{t_{1}, t_{2}} \Subset \Theta$ be a cylinder. By Theorem 3.5, $h$ is subparabolic in $U_{t_{1}, t_{2}}$. Since $h$ is continuous on $\bar{U}_{t_{1}, t_{2}}$, it is also bounded. By Lemma 3.2, we have that $\tilde{u}=\min \left\{u, \max _{\bar{U}_{t_{1}, t_{2}}} h\right\}$ is a bounded superparabolic function. We can thus apply the comparison principle in Theorem 3.6 to conclude that $h \leq \tilde{u} \leq u$ in $U_{t_{1}, t_{2}}$.

The proof for the subparabolic case is similar.

\section{Further Results on Superparabolic Functions}

We continue with a few more results on superparabolic functions that will be needed later on.

The deep result below (Theorem 4.1) completes the relation between superparabolic functions and supersolutions. In particular, a bounded function is superparabolic if and only if it is an lsc-regularized supersolution.

Theorem 4.1. (Kinnunen-Lindqvist [29, Theorems 3.2 and 6.2]) Let $m \geq 1$ and $u$ be superparabolic. Then the following are true:

(a) $u$ is lsc-regularized, and moreover

$$
u(x, t)=\underset{\substack{(y, s) \rightarrow(x, t) \\ s<t}}{\operatorname{ess} \lim \inf } u(y, s)
$$

(b) if $u$ is locally bounded, then $u$ is a supersolution.

Theorems 3.2 and 6.2 of [29] rely on the results about the obstacle problem for the porous medium equation discussed in Lemma 2.18 of the same paper. The main arguments are just sketched, and the interested reader is referred elsewhere for the details. Recently, the obstacle problem for the porous medium equation has been extensively studied in Bögelein-Lukkari-Scheven [14] in a rather general 
framework, and it is not hard to check that [29, Lemma 2.18] can be considered as a special case of [14, Theorem 2.6 and Corollary 2.8].

We will also need the corresponding result for subparabolic functions.

Theorem 4.2. Let $m \geq 1$ and $u$ be subparabolic. Then the following are true:

(a) $u$ is usc-regularized, and moreover

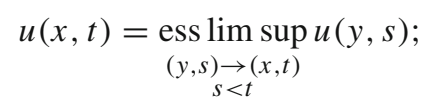

(b) $u$ is a subsolution.

Proof. As Kinnunen-Lindqvist [29] deals also with sign-changing functions, this follows directly by applying Theorem 4.1 to $-u$.

For (b) we do not need to assume that $u$ is locally bounded, as this is automatic for nonnegative subparabolic functions.

The following result completes the picture:

Proposition 4.3. Let $m \geq 1$ and $u$ be a nonnegative function in $\Theta$. Then $u$ is parabolic if and only if it is both sub-and superparabolic in $\Theta$.

Note that a parabolic function is, by Definition 2.1, a continuous solution, whereas sub- and superparabolicity is defined using the quite different Definition 3.1 .

Proof. First assume that $u$ is both sub- and superparabolic. Then, $u$ is continuous. Let $U_{t_{1}, t_{2}} \subset \Theta$ be a $C^{2, \alpha}$-cylinder. By Theorem 2.4, there is $h \in C\left(\bar{U}_{t_{1}, t_{2}}\right)$ which is parabolic in $U_{t_{1}, t_{2}}$ and satisfies $h=u$ on $\partial_{p} U_{t_{1}, t_{2}}$. Since $u$ is superparabolic, $h \leq u$ in $U_{t_{1}, t_{2}}$, and as $u$ is subparabolic, $h \geq u$ in $U_{t_{1}, t_{2}}$, i.e. $u=h$ in $U_{t_{1}, t_{2}}$, and in particular $u$ is parabolic in $U_{t_{1}, t_{2}}$. As being a solution of an equation is a local property, $u$ is parabolic in $\Theta$.

Conversely, assume that $u$ is parabolic. Then $u$ is continuous, and thus $u=$ $u_{*}=u^{*}$. By Theorem 3.5, $u$ is both sub- and superparabolic.

Recall that $\Theta_{T}=\{(x, t) \in \Theta: t<T\}$.

Proposition 4.4. Let $m>0$. Assume that $v$ is a subparabolic function in $\Theta_{T}$ satisfying $v \geq c$ for some $c \geq 0$. Then the function

$$
w(x, t)= \begin{cases}v(x, t), & \text { if }(x, t) \in \Theta_{T}, \\ \limsup _{\Theta_{T} \ni(y, s) \rightarrow(x, t)} v(y, s), & \text { if }(x, t) \in \Theta \text { and } t=T, \\ c, & \text { if }(x, t) \in \Theta \text { and } t>T,\end{cases}
$$

is subparabolic in $\Theta$. 
Proposition 4.5. Let $m>0$. Assume that $v$ is a superparabolic function in $\Theta_{T}$ satisfying $v \leq M$ for some $M<\infty$. Then the function

$$
w(x, t)= \begin{cases}v(x, t), & \text { if }(x, t) \in \Theta_{T}, \\ \liminf _{T \ni(y, s) \rightarrow(x, t)} v(y, s), & \text { if }(x, t) \in \Theta \text { and } t=T, \\ M, & \text { if }(x, t) \in \Theta \text { and } t>T,\end{cases}
$$

is superparabolic in $\Theta$.

The proofs of these two results are similar, we give the proof of the latter one.

Proof. Since $v$ is lower semicontinuous, so is $w$, and it is also bounded. It remains to show the comparison principle. To this end, let $U_{t_{1}, t_{2}} \Subset \Theta$ be a $C^{2, \alpha}$-cylinder, and $h \in C\left(\bar{U}_{t_{1}, t_{2}}\right)$ be parabolic in $U_{t_{1}, t_{2}}$ and such that $h \leq w$ on $\partial_{p} U_{t_{1}, t_{2}}$. In particular $h \leq M$ on $\partial_{p} U_{t_{1}, t_{2}}$, and thus by (the comparison part of) Theorem $2.4, h \leq M$ in $U_{t_{1}, t_{2}}$. Since $v$ is superparabolic in $\Theta_{T}$, we see that $h \leq w$ in $U_{t_{1}, t_{2}}$ if either $t_{2}<T$ or $t_{1} \geq T$.

Assume therefore that $t_{1}<T \leq t_{2}$. Since $w=v \geq h$ in $U_{t_{1}, s}$ for each $s<T$, this holds also in $U_{t_{1}, T}$. If $t_{2}>T$, it follows from the definition of $w$ and the continuity of $h$ that $h \leq w$ in $U \times\{T\}$, and moreover $h \leq M=w$ in $U_{T, t_{2}}$.

Using Theorem 2.5 we can obtain the following convergence result:

Proposition 4.6. Let $m>0$ and $u_{k}$ be an increasing sequence of superparabolic functions in $\Theta$. If $u:=\lim _{k \rightarrow \infty} u_{k}$ is finite in a dense subset of $\Theta$, then $u$ is superparabolic in $\Theta$.

Proof. As the sequence is increasing, $u$ is automatically lower semicontinuous, and thus it is only the comparison principle (iii) that we need to prove. Let $U_{t_{1}, t_{2}} \Subset \Theta$ be a $C^{2, \alpha}$-cylinder, and let $h \in C\left(\bar{U}_{t_{1}, t_{2}}\right)$ be parabolic in $U_{t_{1}, t_{2}}$ and satisfy $h \leq u$ on $\partial_{p} U_{t_{1}, t_{2}}$. Let $h_{j}=\left(\left.h\right|_{\partial_{p} U_{t_{1}, t_{2}}}-1 / j\right)_{+}$on $\partial_{p} U_{t_{1}, t_{2}}$ and extend it to $U_{t_{1}, t_{2}}$ as the unique continuous extension which is parabolic in $U_{t_{1}, t_{2}}$, as provided by Theorem 2.4 . It follows from the compactness and the lower semicontinuity that for each $j$ there is $k_{j}$ such that $h_{j} \leq u_{k_{j}}$ on $\partial_{p} U_{t_{1}, t_{2}}$. As $u_{k_{j}}$ is superparabolic, it then follows from the definition that $h_{j} \leq u_{k_{j}} \leq u$ in $U_{t_{1}, t_{2}}$. By Theorem 2.5, $h \leq u$ in $U_{t_{1}, t_{2}}$. Thus $u$ is superparabolic.

For subparabolic functions we have the following result:

Proposition 4.7. Let $m>0$ and $u_{k}$ be a decreasing sequence of subparabolic functions in $\Theta$. Then $u:=\lim _{k \rightarrow \infty} u$ is subparabolic in $\Theta$.

Proof. The proof is almost identical to the proof of Proposition 4.6. However, this time the finiteness is automatic.

Using this we can improve on Proposition 3.3 in Kinnunen-Lindqvist [29] as follows (for nonnegative functions). 
Proposition 4.8. Let $m \geq 1$. If $u_{k}$ is an increasing sequence of supersolutions and $u:=\lim _{k \rightarrow \infty} u$ is locally bounded, then $u$ is a supersolution.

Similarly, if $u_{k}$ is a decreasing sequence of subsolutions, then $u:=\lim _{k \rightarrow \infty} u$ is a subsolution.

Proof. Consider first the case of supersolutions. By Theorem 3.3 we may assume that $u_{k}$ are lsc-regularized. By Theorem 3.5, $u_{k}$ is superparabolic, and thus $u$ is superparabolic, by Proposition 4.6. It then follows that $u$ is a supersolution by Theorem 4.1.

The case for subsolutions is obtained similarly. As before there is no need to assume local boundedness.

We can now also conclude the following result, which we have not seen in the literature, though it might be well known to experts in the field:

Proposition 4.9. Let $m \geq 1$. If $u$ and $v$ are supersolutions, then so is $\min \{u, v\}$. Similarly, if $u$ and $v$ are subsolutions, then so is $\max \{u, v\}$.

To prove this we need the following characterization:

Proposition 4.10. Let $m>0$ and $u: \Theta \rightarrow[0, \infty]$ be a function such that $u^{m} \in$ $L^{2}\left(t_{1}, t_{2} ; W^{1,2}(U)\right)$ whenever $U_{t_{1}, t_{2}} \Subset \Theta$. Then $u$ is a supersolution if and only if $u_{k}:=\min \{u, k\}$ is a supersolution for all $k=1,2, \ldots$

Proof. Assume first that $u$ is a supersolution. Then it follows from DiBenedettoGianazza-Vespri [23, Lemma 3.5.1] that also $u_{k}$ is a supersolution, if $m \geq 1$. For $0<m<1$, this was proved in a slightly different context, and for a wider class of equations, in Bögelein-Duzaar-Gianazza [13, Lemma 3.1].

Conversely, assume that $u_{k}, k=1,2, \ldots$, are supersolutions. Let $U_{t_{1}, t_{2}} \Subset \Theta$ be a cylinder and $\varphi \in C_{0}^{\infty}\left(U_{t_{1}, t_{2}}\right)$ be nonnegative. Then

$$
\begin{aligned}
& \int_{t_{1}}^{t_{2}} \int_{U} \nabla u^{m} \cdot \nabla \varphi d x d t-\int_{t_{1}}^{t_{2}} \int_{U} u \partial_{t} \varphi d x d t \\
& \quad=\lim _{k \rightarrow \infty}\left(\int_{t_{1}}^{t_{2}} \int_{U} \nabla u_{k}^{m} \cdot \nabla \varphi d x d t-\int_{t_{1}}^{t_{2}} \int_{U} u_{k} \partial_{t} \varphi d x d t\right) \geq 0,
\end{aligned}
$$

and thus $u$ is a supersolution.

Using the above characterization we can obtain the following consequence (cf. Theorem 4.1 (b)):

Proposition 4.11. Let $m \geq 1$ and let $u$ be superparabolic in $\Theta$. If $u^{m} \in$ $L^{2}\left(t_{1}, t_{2} ; W^{1,2}(U)\right)$ whenever $U_{t_{1}, t_{2}} \Subset \Theta$, then $u$ is a supersolution in $\Theta$.

In general this kind of regularity does not hold for superparabolic functions, since there are superparabolic functions which are not supersolutions, the Barenblatt solution being perhaps the easiest example, see Kinnunen-Lindqvist [29, p. 148].

Proof. Let $k>0$. By Lemma 3.2, $u_{k}:=\min \{u, k\}$ is superparabolic, and hence a supersolution by Theorem 4.1 (b). It then follows from Proposition 4.10, that $u$ is a supersolution. 
Proof of Proposition 4.9. First, assume that $u$ and $v$ are supersolutions. By Theorem 3.3, we may without loss of generality assume that they are lsc-regularized. It then follows from Theorem 3.5 that they both are superparabolic, and hence by Lemma 3.2, so is $\min \{u, v\}$. Let $U_{t_{1}, t_{2}} \Subset \Theta$. As $u^{m}, v^{m} \in L^{2}\left(t_{1}, t_{2} ; W^{1,2}(U)\right)$, also $\min \{u, v\}^{m} \in L^{2}\left(t_{1}, t_{2} ; W^{1,2}(U)\right)$. Thus it follows from Proposition 4.11 that $\min \{u, v\}$ is a supersolution.

Next, we turn to the case when $u$ and $v$ are subsolutions. By Theorem 3.4, we may assume that they are usc-regularized. It then follows from Theorem 3.5 that they both are subparabolic, and, by Lemma 3.2, so is $\max \{u, v\}$. Finally, by Theorem $4.2(\mathrm{~b}), \max \{u, v\}$ is a subsolution.

\section{Comparison Principles for Sub- and Superparabolic Functions}

In this section we obtain a series of different kinds of comparison principles for sub- and superparabolic functions, which will be important later on. Recall that one such comparison principle has already been obtained for cylinders when $m \geq 1$ in Theorem 3.6. Note that the next two theorems do not require $m \geq 1$.

Theorem 5.1. (Parabolic comparison principle for general sets) Let $m>0$ and $\Theta$ be bounded. Suppose that $u$ is superparabolic and $v$ is subparabolic in $\Theta$. Let $T \in \mathbf{R}$ and assume that

$$
\infty \neq \limsup _{\Theta \ni(y, s) \rightarrow(x, t)} v(y, s)<\liminf _{\Theta \ni(y, s) \rightarrow(x, t)} u(y, s)
$$

for all $(x, t) \in\{(x, t) \in \partial \Theta: t<T\}$. Then $v \leq u$ in $\{(x, t) \in \Theta: t<T\}$.

Remark 5.2. The proof of this comparison principle is very different from the proof of the nonstrict comparison principle in cylinders in Theorem 3.6. Our proof is based on the proof in Björn-Björn-Gianazza-Parviainen [10, Theorem 2.4] for the $p$-parabolic equation (1.2).

Proof of Theorem 5.1. Let $\varepsilon>0$ and

$$
E=\{(x, t) \in \Theta: t \leq T-\varepsilon \text { and } v(x, t)>u(x, t)\} .
$$

By (5.1), together with the compactness of $\{(x, t) \in \partial \Theta: t \leq T-\varepsilon\}$ and the semicontinuity of $u$ and $v$, we conclude that $\bar{E}$ is a compact subset of $\Theta$. We argue by contradiction. Assume that $E \neq \varnothing$, and let

$T_{0}=\inf \{t:(x, t) \in E\}=\min \{t:(x, t) \in \bar{E}\} \quad$ and $\quad K=\left\{(x, t) \in \bar{E}: t=T_{0}\right\}$.

Since $K$ is compact, we can find an open $C^{2, \alpha}$-smooth set $U \subset \mathbf{R}^{n}$ such that

$$
K \Subset U \times\left\{T_{0}\right\} \Subset \Theta,
$$

and thus also $\sigma<T_{0}<\tau$ such that

$$
\{(x, t) \in E: t \leq \tau\} \Subset U \times(\sigma, \tau] \Subset \Theta .
$$


In particular, the parabolic boundary $\partial_{p} U_{\sigma, \tau} \subset \Theta \backslash E$, and hence $v \leq u$ on $\partial_{p} U_{\sigma, \tau}$. (Here we could apply Theorem 3.6, but apart from adding the requirement $m \geq 1$, it would also make this proof less elementary.) Due to the semicontinuity of $u$ and $v$, there is a continuous function $\psi$ on $\partial_{p} U_{\sigma, \tau}$ such that $v \leq \psi \leq u$. By Theorem 2.4, we can find a function $h \in C\left(\bar{U}_{\sigma, \tau}\right)$ which is parabolic in $U_{\sigma, \tau}$ and continuously attains its boundary values $h=\psi$ on $\partial_{p} U_{\sigma, \tau}$.

The comparison principle in the definition of sub/superparabolic functions applied in $U_{\sigma, \tau}$ to $v$ and $h$, and to $u$ and $h$, shows that $v \leq h \leq u$ in $U_{\sigma, \tau}$. Thus, we obtain that $U_{\sigma, \tau} \cap E=\varnothing$, and so $T_{0} \geq \tau$, which gives a contradiction. Hence $E$ must be empty, and letting $\varepsilon \rightarrow 0$ concludes the proof.

A direct consequence of Theorem 5.1 is the following comparison principle, which can be considered as a sort of elliptic version of the comparison principle, since it does not acknowledge the parabolic boundary and uses all boundary points (to prove it just apply Theorem 5.1 with a large enough $T$ ):

Theorem 5.3. (Elliptic-type comparison principle) Let $m>0$ and $\Theta$ be bounded. Suppose that $u$ is superparabolic and $v$ is subparabolic in $\Theta$. If

$$
\infty \neq \limsup _{\Theta \ni(y, s) \rightarrow(x, t)} v(y, s)<\liminf _{\Theta \ni(y, s) \rightarrow(x, t)} u(y, s)
$$

for all $(x, t) \in \partial \Theta$, then $v \leq u$ in $\Theta$.

Both in Theorems 5.1 and 5.3 we would have liked to have nonstrict comparison principles, only assuming nonstrict inequalities in (5.1) and (5.2), but since we cannot add constants to sub/superparabolic functions, we have not been able to achieve this. In fact, this is a well-known problem with the comparison principle, and the nonstrict elliptic comparison principle is known to be equivalent to the fundamental inequality $\underline{P} f \leq \bar{P} f$ between lower and upper Perron solutions, see Definition 6.1 below. Moreover, the parabolic-type and elliptic-type comparison principles in Theorems 5.1 and 5.3 are equivalent, since the former follows from the latter together with Propositions 4.4 and 4.5.

In both comparison principles the conclusion is nonstrict, even though the inequalities in (5.1) and (5.2) are strict. If one knew that $u_{\psi}<u_{\psi+\varepsilon}$, where $\psi \in C\left(\partial_{p} U_{t_{1}, t_{2}}\right)$ is positive and $u_{\psi}$ and $u_{\psi+\varepsilon}$ are as provided by Theorem 2.4 , then a strict inequality could also be concluded, but this seems to be one of the many open questions in the area.

Next, we extend the nonstrict parabolic comparison principle in Theorem 3.6 to unions of bounded cylinders. Note that this improvement also removes the boundedness assumption from Theorem 3.6.

Theorem 5.4. (Parabolic comparison principle for unions of cylinders) Let $m \geq 1$ and $\Theta$ be a finite union of bounded cylinders in $\mathbf{R}^{n+1}$. Suppose that $u$ is superparabolic and $v$ is subparabolic in $\Theta$. Assume that

$$
\infty \neq \limsup _{\Theta \ni(y, s) \rightarrow(x, t)} v(y, s) \leq \liminf _{\Theta \ni(y, s) \rightarrow(x, t)} u(y, s)
$$

for all $(x, t) \in \partial_{p} \Theta$. Then $v \leq u$ in $\Theta$. 
Proof. Assume that $\Theta=\bigcup_{j=1}^{N} U_{t_{j}, s_{j}}^{j}$ and extend $u$ and $v$ to $(x, t) \in \partial_{p} \Theta$, by letting

$$
v(x, t)=\limsup _{\Theta \ni(y, s) \rightarrow(x, t)} v(y, s) \text { and } u(x, t)=\liminf _{\Theta \ni(y, s) \rightarrow(x, t)} u(y, s) .
$$

We argue by contradiction, assuming that $E=\{\xi \in \Theta: v(\xi)>u(\xi)\}$ is nonempty. Let $\tau=\inf \{t:(x, t) \in E\}$ and

$$
S=\left\{t_{1}, s_{1}, t_{2}, s_{2}, \ldots, t_{N}, s_{N}\right\}
$$

We now divide the proof into three cases.

Case 1. $\tau \notin S$ and thus $\min S<\tau<\max S$. Let

$$
t_{0}=\max \{t \in S: t<\tau\} \quad \text { and } \quad s_{0}=\min \{t \in S: t>\tau\} .
$$

Then $t_{0}<\tau<s_{0}, U_{t_{0}, s_{0}}=\left\{(x, t) \in \Theta: t_{0}<t<s_{0}\right\}$ is a cylinder, and $v \leq u$ on $\partial_{p} U_{t_{0}, s_{0}}$. Hence $v \leq u$ in $U_{t_{0}, s_{0}}$ by Theorem 3.6. But this contradicts the fact that $\tau<s_{0}$.

Case 2. $\tau \in S$ but there is no point $(x, \tau) \in E$. In this case we let $t_{0}=\tau$ and proceed as in Case 1 .

Case 3. $\tau \in S$ and there is at least one point $\left(x_{\tau}, \tau\right) \in E$. First, we show that $v$ is bounded. As $v$ is upper semicontinuous and does not take the value $\infty$ at the compact set $\partial_{p} \Theta$, there is $M<\infty$ such that $v<M$ on $\partial_{p} \Theta$. It then follows from Theorem 5.1 that $v \leq M$ in $\Theta$.

Next, since $\left(x_{\tau}, \tau\right) \in E \subset \Theta$, we can find a $C^{2, \alpha}$-cylinder $U_{t^{\prime}, \tau} \Subset \Theta$ with $x_{\tau} \in U$. Then there is a continuous $h:\left\{(x, t) \in \partial U_{t^{\prime}, \tau}: t<\tau\right\}=: A \rightarrow \mathbf{R}$ such that $v \leq h \leq u$ on $A$. As $v$ is bounded, we can choose $h$ to be bounded. We can then iterate Theorem 2.4 on $U_{t^{\prime}, \tau-1 / j}, j=1,2, \ldots$, to find a continuous solution, also called $h$, in $U_{t^{\prime}, \tau}$ which has $h$ as continuous boundary values on $A$. By iterating also Theorem 3.6, we see that $v \leq h \leq u$ in $U_{t^{\prime}, \tau}$. By DiBenedetto-GianazzaVespri [23, Theorem 5.16.1], $h$ has a continuous extension (also called $h$ ) to the top $U \times\{\tau\}$. We then get from Theorems 4.1 and 4.2 that

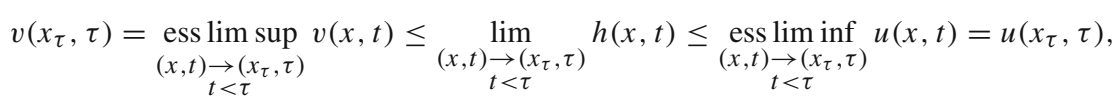

which contradicts the fact that $\left(x_{\tau}, \tau\right) \in E$.

The following lemma is useful when constructing new superparabolic functions:

Lemma 5.5. (Pasting lemma) Let $m \geq 1$ and $G \subset \Theta$ be open. Suppose $u$ and $v$ are superparabolic in $\Theta$ and $G$, respectively. Let

$$
w= \begin{cases}\min \{u, v\} & \text { in } G \\ u & \text { in } \Theta \backslash G\end{cases}
$$

If $\overline{\{(x, t) \in G: v(x, t)<u(x, t)\}} \cap \Theta \subset G$, then $w$ is superparabolic in $\Theta$. 
This is a more restrictive pasting lemma than the one for $p$-parabolic functions in Björn-Björn-Gianazza-Parviainen [10, Lemma 2.9]. As we only have a strict comparison principle in Theorem 5.1, the proof in [10] does not carry over to our situation. If however $u$ is constant, then we can obtain the full pasting lemma. Note that in applications the pasting lemma is often used with a constant "outer" function.

Lemma 5.6. (Pasting lemma) Let $m \geq 1$ and $0 \leq k<\infty$. Suppose $G \subset \Theta$ is open and $v$ is superparabolic in $G$. Define

$$
w= \begin{cases}\min \{k, v\} & \text { in } G, \\ k & \text { in } \Theta \backslash G .\end{cases}
$$

If $w$ is lower semicontinuous, then $w$ is superparabolic in $\Theta$.

Before proving Lemma 5.5, we first show how it can be used to obtain Lemma 5.6.

Proof. Let $k_{j}=(k-1 / j)_{+}$and

$$
w_{j}= \begin{cases}\min \left\{k_{j}, v\right\} & \text { in } G, \\ k_{j} & \text { in } \Theta \backslash G,\end{cases}
$$

$j=1,2, \ldots$. As $w$ is lower semicontinuous, it follows from the local compactness of $\Theta \cap \partial G$ that $\overline{\left\{(x, t) \in G: v(x, t)<k_{j}\right\}} \cap \Theta \subset G$. Hence, by Lemma 5.5, $w_{j}$ is superparabolic. Finally, using Proposition 4.6, $w$ is superparabolic.

Proof of Lemma 5.5. Let us first show that $w$ is lower semicontinuous. This is clear in $G$ and in $\Theta \backslash \bar{G}$. Let $\xi \in \Theta \cap \partial G$. Then, by assumption, $w=u$ in a neighbourhood of $\xi$ and, since $u$ is lower semicontinuous, we conclude that $w$ is lower semicontinuous at $\xi$, i.e. $w$ is lower semicontinuous in $\Theta$.

Since $0 \leq w \leq u, w$ is finite in a dense subset of $\Theta$, and we only have to obtain the comparison principle. Therefore, let $U_{t_{1}, t_{2}} \Subset \Theta$ be a $C^{2, \alpha}$-cylinder, and $h \in C\left(\bar{U}_{t_{1}, t_{2}}\right)$ be parabolic in $U_{t_{1}, t_{2}}$ and such that $h \leq w$ on $\partial_{p} U_{t_{1}, t_{2}}$. Since $h \leq u$ on $\partial_{p} U_{t_{1}, t_{2}}$ and $u$ is superparabolic, we directly have that $h \leq u$ in $U_{t_{1}, t_{2}}$.

Next, let $A=\overline{\left\{(x, t) \in U_{t_{1}, t_{2}} \cap G: v(x, t)<u(x, t)\right\}}$. Then $A \Subset G$ by assumption. Thus, by compactness, we can cover $A$ by a finite number of cylinders $V_{\sigma_{j}, \tau_{j}}^{j}:=V^{j} \times\left(\sigma_{j}, \tau_{j}\right) \Subset G$. Let $\Xi=\bigcup_{j=1}^{m}\left(V_{\sigma_{j}, \tau_{j}}^{j} \cap U_{t_{1}, t_{2}}\right)$, which is a finite union of cylinders. If $\xi \in \partial_{p} \Xi$, then either $\xi \in \partial_{p} U_{t_{1}, t_{2}}$ and thus $h(\xi) \leq w(\xi) \leq v(\xi)$, or $\xi \in U_{t_{1}, t_{2}} \backslash A$ in which case $h(\xi) \leq u(\xi) \leq v(\xi)$. In either case $h \leq v$ on $\partial_{p} \Xi$. Since $h$ is continuous it follows from the comparison principle in Theorem 5.4 that $h \leq v$ in $\Xi$. Thus $h \leq w$ in $U_{t_{1}, t_{2}}$ which shows that $w$ is superparabolic in $\Theta$.

We also need the corresponding pasting lemmas for subparabolic functions. While these are not immediate consequences of the ones for superparabolic functions, the proofs are easy modifications of the proofs for the superparabolic pasting lemmas. We omit the details. 
Lemma 5.7. (Pasting lemma) Let $m \geq 1$ and $G \subset \Theta$ be open. Suppose $u$ and $v$ are subparabolic in $\Theta$ and $G$, respectively. Let

$$
w= \begin{cases}\max \{u, v\} & \text { in } G, \\ u & \text { in } \Theta \backslash G .\end{cases}
$$

If $\overline{\{(x, t) \in G: v(x, t)>u(x, t)\}} \cap \Theta \subset G$, then $w$ is subparabolic in $\Theta$.

Lemma 5.8. (Pasting lemma) Let $m \geq 1$ and $0 \leq k<\infty$. Suppose $G \subset \Theta$ is open and $v$ is subparabolic in G. Define

$$
w= \begin{cases}\max \{k, v\} & \text { in } G, \\ k & \text { in } \Theta \backslash G .\end{cases}
$$

If $w$ is upper semicontinuous, then $w$ is subparabolic in $\Theta$.

\section{The Perron Method and Boundary Regularity}

In Sects. 6-11, $\Theta \subset \mathbf{R}^{n+1}$ is always a bounded open set.

Now we come to the Perron method for (2.1). For us it will be enough to consider Perron solutions for bounded (and nonnegative) functions, so for simplicity we restrict ourselves to this case throughout the paper.

Definition 6.1. Given a bounded $f: \partial \Theta \rightarrow[0, \infty)$, let the upper class $\mathscr{U}_{f}$ be the set of all superparabolic functions $u$ on $\Theta$ such that

$$
\liminf _{\Theta \ni \eta \rightarrow \xi} u(\eta) \geq f(\xi) \text { for all } \xi \in \partial \Theta .
$$

Define the upper Perron solution of $f$ by

$$
\bar{P} f(\xi)=\inf _{u \in \mathscr{U}_{f}} u(\xi), \quad \xi \in \Theta .
$$

Similarly, let the lower class $\mathscr{L}_{f}$ be the set of all subparabolic functions $u$ on $\Theta$ which are bounded above and such that

$$
\limsup _{\Theta \ni \eta \rightarrow \xi} u(\eta) \leq f(\xi) \text { for all } \xi \in \partial \Theta,
$$

and define the lower Perron solution of $f$ by

$$
\underline{P} f(\xi)=\sup _{u \in \mathscr{L}_{f}} u(\xi), \quad \xi \in \Theta
$$

If $\underline{P} f=\bar{P} f$, then $f$ is called resolutive.

Since we only have strict comparison principles in Theorems 5.1 and 5.3, we also introduce strict Perron solutions as follows. 
Definition 6.2. Given a bounded $f: \partial \Theta \rightarrow[0, \infty)$, let the upper class $\tilde{\mathscr{U}}_{f}$ be the set of all superparabolic functions $u$ on $\Theta$ such that

$$
\liminf _{\Theta \ni \eta \rightarrow \xi} u(\eta)>f(\xi) \text { for all } \xi \in \partial \Theta .
$$

Define the upper strict Perron solution of $f$ by

$$
\bar{S} f(\xi)=\inf _{u \in \widetilde{\mathscr{U}}_{f}} u(\xi), \quad \xi \in \Theta .
$$

Similarly, let the lower class $\tilde{\mathscr{L}}_{f}$ be the set of all subparabolic functions $u$ on $\Theta$ which are bounded above and such that

$$
\limsup _{\Theta \ni \eta \rightarrow \xi} u(\eta)<f(\xi) \text { for all } \xi \in \partial \Theta .
$$

Define the lower strict Perron solution of $f$ by

$$
\underline{S} f(\xi)=\sup _{u \in \widetilde{\mathscr{L}}_{f}} u(\xi), \quad \xi \in \Theta
$$

if $\tilde{\mathscr{L}}_{f} \neq \varnothing$, and set $\underline{S} f \equiv 0$ if $\tilde{\mathscr{L}}_{f}=\varnothing$.

Since $\tilde{\mathscr{L}}_{f}=\varnothing$ if, and only if, $f$ takes the value 0 at some boundary point (the constant zero function allowed otherwise is excluded in this case), the lower strict Perron solution is rather restrictive. A possibility would have been to consider signed subparabolic functions in the definition of $\widetilde{\mathscr{L}}_{f}$, which we have refrained from since that would lead into uncharted territory.

Remark 6.3. Observe that the definitions of Perron solutions always depend on the set $\Theta$. To emphasize this dependence, we will at times use the notation $\bar{P}_{\Theta} f, \underline{P}_{\Theta} f, \bar{S}_{\Theta} f$ and $\underline{S}_{\Theta} f$, as well as $\mathscr{U}_{f}(\Theta), \mathscr{L}_{f}(\Theta), \widetilde{\mathscr{U}}_{f}(\Theta)$ and $\widetilde{\mathscr{L}}_{f}(\Theta)$.

It follows from the elliptic-type comparison principle in Theorem 5.3 that $v \leq u$ whenever $u \in \widetilde{\mathscr{U}}_{f}$ and $v \in \mathscr{L}_{f}$. Hence $\underline{S} f \leq \underline{P} f \leq \bar{S} f$ and similarly, $\underline{S} f \leq \bar{P} \bar{f} \leq$ $\bar{S} f$. The inequality $\underline{P} f \leq \bar{P} f$ is only known for finite unions of cylinders, in which case it follows directly from the parabolic comparison principle in Theorem 5.4.

A key question in the theory is whether in general $\underline{P} f=\bar{P} f$. If this happens, the boundary data $f$ are called resolutive. A recent resolutivity result from Kinnunen-Lindqvist-Lukkari [30, Theorem 5.1] shows that continuous functions are resolutive on general cylinders when $m>1$. In Theorem 11.2 below we generalize this result to certain unions of cylinders. For $m=1$ (i.e. for the heat equation) resolutivity of continuous functions holds in arbitrary bounded open sets, see e.g. Watson [43, Theorem 8.26].

Note that we have elliptic-type boundary conditions on the full boundary, not just on the possibly smaller parabolic boundary, whenever it is defined. This is similar to the case of the $p$-parabolic equation (1.2) in Björn-BjörnGianazza-Parviainen [10]. Nevertheless, the following result is true (recall that $\left.\Theta_{T}=\{(x, t) \in \Theta: t<T\}\right)$ : 
Lemma 6.4. Let $m>0, T \in \mathbf{R}$ and suppose that $f: \partial \Theta \rightarrow[0, \infty)$ is bounded. Then

$$
\bar{P}_{f}=\bar{P}^{T} f \text { in } \Theta_{T},
$$

where $\bar{P}^{T} f$ is the infimum of all superparabolic functions in $\Theta_{T}$ such that

$$
\liminf _{\Theta_{T} \ni \eta \rightarrow \xi} u(\eta) \geq f(\xi) \text { for all } \xi=(x, t) \in \partial \Theta \text { with } t<T .
$$

Similar identity holds for $\underline{P} f, \bar{S} f$ and, when $f$ is bounded away from 0 , also for $\underline{S} f$, with obvious modifications in the definitions.

Applying Lemma 6.4 to both $\Theta$ and $\Theta_{T}$ immediately gives the following corollary:

Corollary 6.5. Let $m>0, T \in \mathbf{R}$ and suppose that $f: \partial \Theta \cup \partial \Theta_{T} \rightarrow[0, \infty)$ is bounded. Then

$$
\bar{P}_{\Theta_{T}} f=\bar{P}_{\Theta} f, \quad \bar{S}_{\Theta_{T}} f=\bar{S}_{\Theta} f \quad \text { and } \quad \underline{P}_{\Theta_{T}} f=\underline{P}_{\Theta} f \quad \text { in } \Theta_{T} .
$$

If $f$ is, in addition, bounded away from 0 then also $\underline{S}_{\Theta_{T}} f=\underline{S}_{\Theta} f$ in $\Theta_{T}$.

Remark 6.6. Note that the set $\{\xi=(x, t) \in \partial \Theta: t<T\}$ in the definition of $\bar{P}^{T} f$ is in general not compact.

If $\Theta=U_{t_{1}, t_{2}}$ is a cylinder, then the parabolic boundary is included in the full boundary and contains the above set defining $\bar{P}^{T} f$. Also the corresponding classes of admissible superparabolic functions are included in each other. From this we conclude that the Perron solution using only the parabolic boundary $\partial_{p} U_{t_{1}, t_{2}}$ lies between the two solutions $\bar{P} f$ and $\bar{P}^{T} f$, and thus coincides with them.

If $\Theta$ is a finite union of cylinders (and thus the parabolic boundary is defined), the situation is less clear, unless the boundary points not belonging to the parabolic boundary are at the same time, in which case the above argument applies.

Proof of Lemma 6.4. The inequality $\bar{P} f \geq \bar{P}^{T} f$ is obvious, since the restrictions of functions from $\mathscr{U}_{f}$ are admissible in the definitions of $\bar{P}^{T} f$. Conversely, let $\varepsilon>0$ and $u$ be admissible in the definition of $\bar{P}^{T} f$. For $M=\sup _{\partial \Theta} f+1$, let

$$
v(x, t)= \begin{cases}M, & \text { if }(x, t) \in \Theta \text { and } t>T-\varepsilon, \\ \min \{u(x, t), M\}, & \text { if }(x, t) \in \Theta \text { and } t \leq T-\varepsilon .\end{cases}
$$

By Lemma 3.2 and Proposition 4.5, $v$ is superparabolic in $\Theta$ and thus $v \in \mathscr{U}_{f}$. Taking infimum over all $u$ shows that $\bar{P} f(x, t) \leq \bar{P}^{T} f(x, t)$ when $t<T-\varepsilon$. Letting $\varepsilon \rightarrow 0$, yields $\bar{P} f \leq \bar{P}^{T} f$ in $\Theta_{T}$. The identities for $\underline{P} f, \bar{S} f$ and $\underline{S} f$ are proved similarly, possibly replacing Proposition 4.5 by Proposition 4.4 .

Theorem 6.7. Let $m \geq 1$ and suppose that $f: \partial \Theta \rightarrow[0, \infty)$ is bounded. Then $\underline{P} f, \bar{P} f, \underline{S} f$ and $\bar{S} f$ are parabolic, and in particular they are all continuous. 
Proof. For $\underline{P} f$ and $\bar{P} f$ in cylinders this is Theorem 4.6 in Kinnunen-LindqvistLukkari [30] but since everything is local this is true in arbitrary sets, as they in fact mention in [30, p. 2960]. For $m=1$, see e.g. Watson [43].

The proofs carry over essentially verbatim to $\underline{S} f$ and $\bar{S} f$.

Since we cannot add constants to solutions of the porous medium equation, unlike in the elliptic and $p$-parabolic cases, the boundary regularity might a priori depend on the value of the boundary function at that point, and could also be different from above and below. We are therefore led to the following definitions:

Definition 6.8. A boundary point $\xi_{0} \in \partial \Theta$ is upper regular with respect to $\Theta$ if

$$
\limsup _{\Theta \ni \xi \rightarrow \xi_{0}} \bar{P} f(\xi) \leq f\left(\xi_{0}\right)
$$

whenever $f: \partial \Theta \rightarrow(0, \infty)$ is positive and continuous.

Similarly, $\xi_{0}$ is lower regular for positive (nonnegative) boundary data with respect to $\Theta$ if

$$
\liminf _{\Theta \ni \xi \rightarrow \xi_{0}} \underline{P} f(\xi) \geq f\left(\xi_{0}\right)
$$

whenever $f: \partial \Theta \rightarrow[0, \infty)$ is positive (nonnegative) and continuous.

Finally, we say that $\xi_{0}$ is regular for positive (nonnegative) boundary data if it is both upper regular and lower regular for positive (nonnegative) data.

We will often omit the explicit reference to $\Theta$, whenever no confusion may arise. The following result is an elementary but useful tool.

Proposition 6.9. Let $m>0$ and $\xi_{0} \in \partial \Theta$. Then the following are true:

(a) If $f: \partial \Theta \rightarrow[0, \infty)$ is bounded and continuous at $\xi_{0}$, and $\xi_{0}$ is upper regular, then

$$
\limsup _{\Theta \ni \xi \rightarrow \xi_{0}} \bar{P} f(\xi) \leq \limsup _{\Theta \ni \xi \rightarrow \xi_{0}} \bar{S} f(\xi) \leq f\left(\xi_{0}\right) .
$$

(b) If $f: \partial \Theta \rightarrow[0, \infty)$ is bounded and continuous at $\xi_{0}$, and $\xi_{0}$ is lower regular for nonnegative boundary data, then

$$
\liminf _{\Theta \ni \xi \rightarrow \xi_{0}} \underline{P} f(\xi) \geq f\left(\xi_{0}\right)
$$

(c) If $f: \partial \Theta \rightarrow(0, \infty)$ is bounded, bounded away from 0 and continuous at $\xi_{0}$, and $\xi_{0}$ is lower regular for positive boundary data, then

$$
\liminf _{\Theta \ni \xi \rightarrow \xi_{0}} \underline{P} f(\xi) \geq \liminf _{\Theta \ni \xi \rightarrow \xi_{0}} \underline{S} f(\xi) \geq f\left(\xi_{0}\right) .
$$

(d) If $f: \partial \Theta \rightarrow(0, \infty)$ is bounded from above, bounded away from 0 and continuous at $\xi_{0}$, and $\xi_{0}$ is regular for positive boundary data, then

$$
\lim _{\Theta \ni \xi \rightarrow \xi_{0}} \underline{S} f(\xi)=\lim _{\Theta \ni \xi \rightarrow \xi_{0}} \underline{P} f(\xi)=\lim _{\Theta \ni \xi \rightarrow \xi_{0}} \bar{P} f(\xi)=\lim _{\Theta \ni \xi \rightarrow \xi_{0}} \bar{S} f(\xi)=f\left(\xi_{0}\right) .
$$


Proof. (b) Find $\tilde{f} \in C(\partial \Theta)$ so that $0 \leq \tilde{f} \leq f$ and $\tilde{f}\left(\xi_{0}\right)=f\left(\xi_{0}\right)$. Then

$$
\liminf _{\Theta \ni \xi \rightarrow \xi_{0}} \underline{P} f(\xi) \geq \liminf _{\Theta \ni \xi \rightarrow \xi_{0}} \underline{P} \tilde{f}(\xi) \geq \tilde{f}\left(\xi_{0}\right)=f\left(\xi_{0}\right) .
$$

(c) First of all, for any $\varepsilon>0$ small enough, we can find $\tilde{f} \in C(\partial \Theta)$ so that $2 \varepsilon \leq \tilde{f} \leq f$ on $\partial \Theta$ and $\tilde{f}\left(\xi_{0}\right)=f\left(\xi_{0}\right)$. Now $\tilde{f}-\varepsilon$ is positive on $\partial \Theta$, and thus

$$
\liminf _{\Theta \ni \xi \rightarrow \xi_{0}} \underline{S} f(\xi) \geq \liminf _{\Theta \ni \xi \rightarrow \xi_{0}} \underline{P}(\tilde{f}-\varepsilon)(\xi) \geq \tilde{f}\left(\xi_{0}\right)-\varepsilon=f\left(\xi_{0}\right)-\varepsilon .
$$

Letting $\varepsilon \rightarrow 0$ shows the second inequality, while the first one follows directly from the inequality $\underline{P} f \geq \underline{S} f$.

(a) This is shown in the same way as (c), using a continuous function $\tilde{f} \geq f+\varepsilon$ such that $\tilde{f}\left(\xi_{0}\right)=f\left(\xi_{0}\right)+\varepsilon$. $\bar{S} f$.

(d) This follows from (a) and (c) and the inequalities $\underline{S} f \leq \bar{P} f$ and $\underline{P} f \leq$

The following are direct consequences of Proposition 6.9:

(i) Upper regularity is the same for positive and nonnegative boundary data, which is the reason why we did not define them separately. We do not know if such an equivalence holds for lower regularity.

(ii) Upper regularity can equivalently be defined using the upper strict Perron solution $\bar{S}$. Similarly, lower regularity for positive boundary data can be defined using the lower strict Perron solution $\underline{S}$.

It seems that upper regularity is easier to handle. At this point it is far from clear whether upper and lower regularity are equivalent or not, nor if one may imply the other. One can also ask whether (upper/lower) regularity at one level, i.e. for one given boundary value $c \geq 0$ at $\xi_{0}$, is equivalent to regularity at other levels, and also if the growth of the functions plays a role for the regularity.

In the next two sections we are going to show that regularity for positive boundary data can be characterized by the existence of two two-parameter families of barriers, one family from above and one from below. Whether all barriers are really needed to guarantee regularity is far from obvious, but hopefully future research will be able to clarify this.

However, it may be worth to compare with the situation for the $p$-parabolic equation (1.2) for which regularity was characterized using one one-parameter family in Björn-Björn-Gianazza-Parviainen [10, Theorem 3.3]. (The crucial difference here necessitating two two-parameter families instead of one one-parameter family is the fact that we can neither change sign nor add constants.) In BjörnBjörn-Gianazza [9, Proposition 1.2] it was shown that one barrier is not enough to characterize regularity for the $p$-parabolic equation, at least not for $p<2$, but with one barrier one gets regularity for boundary data $f$ not growing too fast, see [9, Proposition 5.1].

For the heat equation one barrier is enough, as was first shown by Bauer [8, Theorems 30 and 31] for general domains.

We end the section with the following result: 
Proposition 6.10. Let $\xi_{0} \in \partial \Theta$ and $a>0$. Assume that $m>0, m \neq 1$. Then $\xi_{0}$ is upper/lower regular for positive (nonnegative) boundary data for the porous medium equation (2.1) if and only if it is upper/lower regular for positive (nonnegative) boundary data for the multiplied equation

$$
\partial_{t} u=a \Delta u^{m} .
$$

For $m=1$ it is well-known that this is false, by e.g. the Petrovskii criterion [38, 39], and our proof breaks down in this case.

Proof. If $u$ is a sub/supersolution of (6.1), then $v=a^{1 /(m-1)} u$ is a sub/supersolution of the porous medium equation (2.1). The whole theory can now equivalently be developed for the equation (6.1) and the upper/lower regularity becomes equivalent.

Since the space $\mathbf{R}^{n+1}$ is homogeneous, one can translate the equation and still have the same regularity. Thus, without loss of generality, we will sometimes assume that the boundary point under consideration is the origin $\xi_{0}=(0,0)$.

\section{Barrier Characterization of Upper Regularity}

Definition 7.1. A family of functions $w_{c, k}$, with $c \in \mathbf{Q}_{+}$and $k=1,2, \ldots$, is an upper barrier family in $\Theta$ at $\xi_{0}=\left(x_{0}, t_{0}\right) \in \partial \Theta$ if for each $c \in \mathbf{Q}_{+}$and $k$,

(a) $w_{c, k}: \Theta \rightarrow[c, \infty)$ is superparabolic;

(b) $\lim _{\Theta \ni \zeta \rightarrow \xi_{0}} w_{c, k}(\zeta)=c$;

(c) there is $j=j(c, k) \in \mathbf{N}$ such that

$$
\liminf _{\Theta \ni \zeta \rightarrow(x, t)} w_{c, j}(\zeta) \geq c+k
$$

for all $(x, t) \in \partial \Theta$ with $\left|x-x_{0}\right|+\left|t-t_{0}\right| \geq 1 / k$.

Here $\mathbf{Q}_{+}=\{x \in \mathbf{Q}: x>0\}$.

Theorem 7.2. Let $m \geq 1$ and $\xi_{0} \in \partial \Theta$. Then the following are equivalent:

(1) $\xi_{0}$ is upper regular;

(2) there is an upper barrier family at $\xi_{0}$;

(3) there is an upper barrier family at $\xi_{0}$ consisting of parabolic functions satisfying $(c)$ in Definition 7.1 with $(x, t) \in \partial \Theta$ replaced by $(x, t) \in \bar{\Theta}$.

Proof. We assume, without loss of generality, that $\xi_{0}=(0,0)$.

$(2) \Rightarrow(1)$ Let $\left\{w_{c, k}\right\}$ be an upper barrier family at $\xi_{0}$.

Assume that $f: \partial \Theta \rightarrow(0, \infty)$ is continuous. Let $c \in \mathbf{Q}_{+}$be such that $c>f\left(\xi_{0}\right)$. Then find $k \geq \sup _{\partial \Theta} f$ such that $f(x, t)<c$ whenever $|x|+|t|<1 / k$. Let $j=j(c, k)$ be as given in Definition 7.1(c). Since $w_{c, j} \geq c$, we see that

$$
\liminf _{\Theta \ni \zeta \rightarrow(x, t)} w_{c, j}(\zeta)>f(x, t) \text { for all }(x, t) \in \partial \Theta
$$


This implies that $w_{c, j} \in \mathscr{U}_{f}$, and thus $\bar{P} f \leq w_{c, j}$ in $\Theta$. Consequently,

$$
\limsup _{\Theta \ni \zeta \rightarrow \xi_{0}} \bar{P} f(\zeta) \leq \limsup _{\Theta \ni \zeta \rightarrow \xi_{0}} w_{c, j}(\zeta)=c .
$$

Since this holds for all rational $c>f\left(\xi_{0}\right)$, we conclude that

$$
\limsup _{\Theta \ni \zeta \rightarrow \xi_{0}} \bar{P} f(\zeta) \leq f\left(\xi_{0}\right)
$$

and thus $\xi_{0}$ is upper regular.

(1) $\Rightarrow$ (3) Assume that $\xi \in \partial \Theta$ is upper regular. Given $c \in \mathbf{Q}_{+}$and $j=1,2, \ldots$, we let

$$
\psi_{c, j}(x, t)=\left(c^{m}+j|x|^{2}+j b t^{2}\right)^{1 / m} \text { for }(x, t) \in \mathbf{R}^{n+1},
$$

where $b=m c^{m-1} / \operatorname{diam} \Theta$. Note that $\psi_{c, j} \geq c$. Direct calculations show that

$$
\partial_{t} \psi_{c, j}=\frac{2 j b t}{m}\left(c^{m}+j|x|^{2}+j b t^{2}\right)^{-1+1 / m} \leq \frac{2 j b t}{m c^{m-1}}
$$

and

$$
\Delta \psi_{c, j}^{m}=j \Delta|x|^{2}=2 j \operatorname{div} x=2 j n \geq 0 .
$$

Hence

$$
\partial_{t} \psi_{c, j}-\Delta \psi_{c, j}^{m} \leq 2 j\left(\frac{b t}{m c^{m-1}}-n\right) \leq 0,
$$

which implies by Theorem 3.5 that $\psi_{c, j}$ is subparabolic in $\Theta$. By Theorem 6.7 the function

$$
w_{c, j}:=\underline{P} \psi_{c, j}
$$

is parabolic. Since $\psi_{c, j}$ is subparabolic, it belongs to $\mathscr{L}_{\psi_{c, j}}$. Therefore, by definition, we get that $w_{c, j} \geq \psi_{c, j} \geq c$, and so (a) in Definition 7.1 holds. As $\xi_{0}$ is upper regular, we also obtain using Proposition 6.9 that

$$
\limsup _{\Theta \ni \xi \rightarrow \xi_{0}} w_{c, j} \leq \limsup _{\Theta \ni \xi \rightarrow \xi_{0}} \bar{S} \psi_{c, j}=c
$$

and thus $\lim _{\Theta \ni \xi \rightarrow \xi_{0}} w_{c, j}=c$, giving (b) in Definition 7.1. By the form of $w_{c, j}$ it follows that also (c) in Definition 7.1 is satisfied, and thus the functions $w_{c, j}$ form an upper barrier family of the type required in (3).

(3) $\Rightarrow(2)$ This is trivial.

The following useful restriction result is a direct consequence of the barrier characterization in Theorem 7.2(3):

Corollary 7.3. Let $m \geq 1$ and $G \subset \Theta$ be open. Suppose that $\xi_{0} \in \partial \Theta \cap \partial G$. If $\xi_{0}$ is upper regular with respect to $\Theta$, then it is upper regular with respect to $G$. 
Another consequence of the barrier characterization is that upper regularity is a local property.

Proposition 7.4. Let $m \geq 1$ and $\xi_{0} \in \partial \Theta$, and suppose that $B$ is a ball containing $\xi_{0}$. Then $\xi_{0}$ is upper regular with respect to $\Theta$ if and only if it is upper regular with respect to $B \cap \Theta$.

Proof. Corollary 7.3 shows that if $\xi_{0}$ is upper regular with respect to $\Theta$, then it is also upper regular with respect to $B \cap \Theta$. It remains to show the converse direction.

By Theorem 7.2 we have an upper barrier family $w_{c, j}$ in $B \cap \Theta$. Let $k_{0}$ be large enough so that $1 / k_{0}<\operatorname{dist}\left(\xi_{0}, \partial B\right)$ and let $j\left(c, k_{0}\right)$ be as in Definition $7.1(\mathrm{c})$. Define

$$
w_{c, k}^{\prime}= \begin{cases}\min \left\{w_{c, j(c, k)}, c+k\right\} & \text { in } B \cap \Theta, \\ c+k & \text { in } \Theta \backslash B,\end{cases}
$$

for $k \geq k_{0}$ and $w_{c, k}^{\prime}=w_{c, k_{0}}^{\prime}$ for $k<k_{0}$. By the pasting lemma 5.6 the function $w_{c, k}^{\prime}$ is superparabolic, and thus $\left\{w_{c, k}^{\prime}\right\}$ is an upper barrier family in $\Theta$. This implies that $\xi_{0}$ is upper regular with respect to $\Theta$.

\section{Barrier Characterization of Lower Regularity for Positive Boundary Data}

Definition 8.1. A family of functions $w_{c, k}$, with $c \in \mathbf{Q}_{+}$and $k=1,2, \ldots$, is a lower barrier family for positive boundary data in $\Theta$ at the point $\xi_{0}=\left(x_{0}, t_{0}\right) \in \partial \Theta$ if for each $c \in \mathbf{Q}_{+}$and $k$,

(a) $w_{c, k}: \Theta \rightarrow[0, c]$ is subparabolic;

(b) $\lim _{\Theta \ni \zeta \rightarrow \xi_{0}} w_{c, k}(\zeta)=c$;

(c) there is $j=j(c, k) \geq k$ such that

$$
\limsup _{\Theta \ni \zeta \rightarrow(x, t)} w_{c, j}(\zeta) \leq \frac{1}{k}
$$

for all $(x, t) \in \partial \Theta$ with $\left|x-x_{0}\right|+\left|t-t_{0}\right| \geq 1 / k$.

Lemma 8.2. Let $m>0$. Assume that $u$ is a supersolution (subsolution) in $\Theta \backslash$ $(E \times \mathbf{R})$, where $E \subset \mathbf{R}^{n}$ is a set of zero capacity such that $\Theta \backslash(E \times \mathbf{R})$ is open. If $u^{m} \in L^{2}\left(t_{1}, t_{2} ; W^{1,2}(U)\right)$ for every cylinder $U_{t_{1}, t_{2}} \Subset \Theta$, then $u$ is a supersolution (subsolution) in $\Theta$ as well.

For the definition of capacity, see (11.1) below.

Proof. We consider supersolutions. The proof for subsolutions is similar. Let $U_{t_{1}, t_{2}} \Subset \Theta$ and $\varphi \in C_{0}^{\infty}\left(U_{t_{1}, t_{2}}\right)$ be arbitrary. Since $E$ has zero capacity, there exist $\eta_{j} \in C_{0}^{\infty}\left(\mathbf{R}^{n}\right)$ such that $0 \leq \eta_{j} \leq 1$ in $\mathbf{R}^{n}, \eta_{j}=1$ in an open neighbourhood of $E$ and $\left\|\eta_{j}\right\|_{W^{1,2}\left(\mathbf{R}^{n}\right)}<1 / j, j=1,2, \ldots$ Set $\varphi_{j}(x, t)=\left(1-\eta_{j}(x)\right) \varphi(x, t)$. 
Then $\varphi_{j} \in C_{0}^{\infty}\left(U_{t_{1}, t_{2}} \backslash(E \times \mathbf{R})\right)$. Inserting $\partial_{t} \varphi_{j}=\partial_{t} \varphi-\eta_{j} \partial_{t} \varphi$ and $\nabla \varphi_{j}=$ $\nabla \varphi-\left(\eta_{j} \nabla \varphi+\varphi \nabla \eta_{j}\right)$ into (2.2) gives

$$
\begin{aligned}
& \int_{t_{1}}^{t_{2}} \int_{U} \nabla u^{m} \cdot \nabla \varphi d x d t-\int_{t_{1}}^{t_{2}} \int_{U} u \partial_{t} \varphi d x d t \\
& \quad \geq \int_{t_{1}}^{t_{2}} \int_{U} \nabla u^{m} \cdot\left(\eta_{j} \nabla \varphi+\varphi \nabla \eta_{j}\right) d x d t-\int_{t_{1}}^{t_{2}} \int_{U} u \eta_{j} \partial_{t} \varphi d x d t .
\end{aligned}
$$

Since $\varphi \in C_{0}^{\infty}\left(U_{t_{1}, t_{2}}\right)$, there exists $M<\infty$ such that $|\varphi|,|\nabla \varphi|,\left|\partial_{t} \varphi\right| \leq M$ on $U_{t_{1}, t_{2}}$ and hence the Cauchy-Schwarz inequality implies that the right-hand side in the last inequality is majorized (in absolute value) by

$$
3 M\left(\int_{t_{1}}^{t_{2}} \int_{U}\left(\left|\nabla u^{m}\right|^{2}+|u|^{2}\right) d x d t\right)^{1 / 2}\left(\int_{t_{1}}^{t_{2}} \int_{U}\left(\left|\nabla \eta_{j}\right|^{2}+\left|\eta_{j}\right|^{2}\right) d x d t\right)^{1 / 2} .
$$

By assumption, the first factor is bounded while the last factor equals

$$
\left(t_{2}-t_{1}\right)^{1 / 2}\left\|\eta_{j}\right\|_{W^{1,2}\left(\mathbf{R}^{n}\right)}
$$

and tends to zero as $j \rightarrow \infty$. Thus, the left-hand side in (2.2) is nonnegative for every $\varphi \in C_{0}^{\infty}\left(U_{t_{1}, t_{2}}\right)$, which concludes the proof.

Theorem 8.3. Let $m \geq 1$ and $\xi_{0} \in \partial \Theta$. Then the following are equivalent:

(1) $\xi_{0}$ is lower regular for positive boundary data;

(2) there is a lower barrier family for positive boundary data at $\xi_{0}$;

(3) there is a lower barrier family for positive boundary data at $\xi_{0}$ consisting of parabolic functions satisfying $(c)$ in Definition 8.1 with $(x, t) \in \partial \Theta$ replaced by $(x, t) \in \bar{\Theta}$.

Proof. We assume, without loss of generality, that $\xi_{0}=(0,0)$.

The proof of $(2) \Rightarrow(1)$ is similar to that of Theorem 7.2 and we omit the details.

(3) $\Rightarrow(2)$ This is trivial.

(1) $\Rightarrow$ (3) Assume that $\xi_{0} \in \partial \Theta$ is lower regular for positive boundary data.

To construct a lower barrier family for positive boundary data at $\xi_{0}$, let $0<\alpha<$ $\gamma<1 / m$ and $d=2+\log \operatorname{diam} \Theta$. Given $c \in \mathbf{Q}_{+}$and $j=1,2, \ldots$, we define for $(x, t) \in \Theta$,

$$
v_{c, j}(x, t)=\left\{\begin{array}{ll}
c^{-1 / \gamma}+j^{\alpha} t^{2}+\frac{j}{d-\log |x|}, & x \neq 0, \\
c^{-1 / \gamma}+j^{\alpha} t^{2}, & x=0,
\end{array} \quad \text { and } \quad \psi_{c, j}=v_{c, j}^{-\gamma} .\right.
$$

Note that $0<\psi_{c, j} \leq c$. Assume that $x \neq 0$ for the moment. Direct calculations show that $\partial_{t} v_{c, j}=2 j^{\alpha} t$,

$$
\nabla v_{c, j}=\frac{j x}{|x|^{2}(d-\log |x|)^{2}}
$$

and

$$
\Delta v_{c, j}=\frac{j[(n-2)(d-\log |x|)+2]}{|x|^{2}(d-\log |x|)^{3}} \geq \frac{2 j}{|x|^{2}(d-\log |x|)^{3}},
$$


since $n \geq 2$. Moreover, we have

$$
\begin{aligned}
& \partial_{t} \psi_{c, j}=-\gamma v_{c, j}^{-\gamma-1} \partial_{t} v_{c, j}, \\
& \nabla \psi_{c, j}^{m}=\nabla v_{c, j}^{-\gamma m}=-\gamma m v_{c, j}^{-\gamma m-1} \nabla v_{c, j},
\end{aligned}
$$

and thus

$$
\Delta \psi_{c, j}^{m}=-\gamma m v_{c, j}^{-\gamma m-1} \Delta v_{c, j}+\gamma m(\gamma m+1) v_{c, j}^{-\gamma m-2}\left|\nabla v_{c, j}\right|^{2} .
$$

It follows that

$$
\begin{aligned}
\partial_{t} \psi_{c, j}-\Delta \psi_{c, j}^{m}= & \gamma m v_{c, j}^{-\gamma m-2}\left[v_{c, j} \Delta v_{c, j}-(\gamma m+1)\left|\nabla v_{c, j}\right|^{2}\right. \\
& \left.-m^{-1} v_{c, j}^{1+\gamma(m-1)} \partial_{t} v_{c, j}\right] .
\end{aligned}
$$

This will be nonnegative if

$$
I:=\frac{2 j v_{c, j}}{|x|^{2}(d-\log |x|)^{3}}-\frac{(\gamma m+1) j^{2}}{|x|^{2}(d-\log |x|)^{4}}-\frac{2}{m} v_{c, j}^{1+\gamma(m-1)} j^{\alpha} t \geq 0 .
$$

Since $v_{c, j} \geq j /(d-\log |x|)$ and $m \geq 1$, we obtain

$$
\begin{aligned}
I & \geq \frac{2 j v_{c, j}}{|x|^{2}(d-\log |x|)^{3}}-\frac{(\gamma m+1) j v_{c, j}}{|x|^{2}(d-\log |x|)^{3}}-2 v_{c, j}^{1+\gamma(m-1)} j^{\alpha} t \\
& =v_{c, j}\left(\frac{(1-\gamma m) j}{|x|^{2}(d-\log |x|)^{3}}-2 v_{c, j}^{\gamma(m-1)} j^{\alpha} t\right) .
\end{aligned}
$$

A straightforward calculation shows that $\rho \mapsto \rho^{2}(d-\log \rho)^{3}$ is increasing on $[0, \operatorname{diam} \Theta]$, which together with $v_{c, j} \leq j\left(c^{-1 / \gamma}+(\operatorname{diam} \Theta)^{2}+1\right)$ and the choice of $d$ yields

$$
I \geq v_{c, j}\left(\frac{(1-\gamma m) j}{8(\operatorname{diam} \Theta)^{2}}-2 j^{\alpha+\gamma(m-1)}\left(c^{-1 / \gamma}+(\operatorname{diam} \Theta)^{2}+1\right)^{\gamma(m-1)} \operatorname{diam} \Theta\right) .
$$

Since $\alpha<\gamma<1 / m$, we have $\alpha+\gamma(m-1)<1$ and it follows that $I \geq 0$ for sufficiently large $j$. Thus, by Theorem 3.5 we see that $\psi_{c, j}$ is superparabolic in $\{(x, t) \in \Theta: x \neq 0\}$ for such $j$. By (8.1) and (8.2), $\nabla \psi_{c, j}^{m} \in L^{2}\left(t_{1}, t_{2} ; W^{1,2}(U)\right)$ whenever $U_{t_{1}, t_{2}} \Subset \Theta$. Hence Lemma 8.2 shows that $\psi_{c, j}$ is superparabolic in $\Theta$ for sufficiently large $j$.

Set $w_{c, j}=\bar{P} \psi_{c, j}$. Then $w_{c, j}$ is parabolic, by Theorem 6.7. Since $\psi_{c, j}$ is superparabolic, it belongs to $\mathscr{U}_{\psi_{c, j}}$, and thus $w_{c, j} \leq \psi_{c, j} \leq c$. By the lower regularity of $\xi_{0}$ for positive boundary data and Proposition 6.9(c), we also have

$$
\liminf _{\Theta \ni \zeta \rightarrow \xi_{0}} w_{c, j}(\zeta) \geq \liminf _{\Theta \ni \zeta \rightarrow \xi_{0}} \underline{S} \psi_{c, j}(\zeta)=\psi_{c, j}\left(\xi_{0}\right)=c,
$$

and thus $\lim _{\Theta \ni \zeta \rightarrow \xi_{0}} w_{c, j}(\zeta)=c$. By the form of $w_{c, j}$ it follows that (c) is satisfied, and thus (after renumbering) the functions $w_{c, j}$ form a lower barrier family for positive boundary data of the type required in (3). 
As in the case of upper regularity we have two results. The first is a direct consequence of the barrier characterization in Theorem 8.3(3), whereas the proof of the second is similar to the proof of Proposition 7.4; we omit the details.

Corollary 8.4. Let $m \geq 1$ and $G \subset \Theta$ be open. Suppose that $\xi_{0} \in \partial \Theta \cap \partial G$. If $\xi_{0}$ is lower regular for positive boundary data with respect to $\Theta$, then it is lower regular for positive boundary data with respect to $G$.

Proposition 8.5. Let $m \geq 1$ and $\xi_{0} \in \partial \Theta$, and suppose that $B$ is a ball containing $\xi_{0}$. Then $\xi_{0}$ is lower regular for positive boundary data with respect to $\Theta$ if and only if it is lower regular for positive boundary data with respect to $B \cap \Theta$.

\section{Earliest Points are Always Regular}

To make the notation easier, we consider regularity of the origin. Recall that

$$
\Theta_{-}=\{(x, t) \in \Theta: t<0\} \text { and } \Theta_{+}=\{(x, t) \in \Theta: t>0\} .
$$

Proposition 9.1. Let $m \geq 1$ and $\xi_{0}=(0,0) \in \partial \Theta$. If $\xi_{0} \notin \partial \Theta_{-}$, which in particular holds if $\Theta_{-}$is empty, then $\xi_{0}$ is regular for positive boundary data.

Proof. By Propositions 7.4 and 8.5 we may assume that $\Theta_{-}=\varnothing$.

First, we turn to upper regularity and let $c \in \mathbf{Q}_{+}$be arbitrary. Let

$$
w_{c, j}=\left(c^{m}+j|x|^{2}+j^{2 m-1} t\right)^{1 / m} \text { and } \delta=\max \{\operatorname{diam} \Theta, 1\} .
$$

Then, as $1 / m-1<0$,

$$
\partial_{t} w_{c, j}=\frac{j^{2 m-1}}{m}\left(c^{m}+j|x|^{2}+j^{2 m-1} t\right)^{1 / m-1} \geq \frac{j^{2 m-1}}{m}\left(c^{m}+2 j^{2 m-1} \delta^{2}\right)^{1 / m-1}
$$

and $\Delta w_{c, j}^{m}=j \Delta|x|^{2}=2 j n$. We want to have

$$
\partial_{t} w_{c, j}-\Delta w_{c, j}^{m} \geq \frac{j^{2 m-1}}{m}\left(c^{m}+2 j^{2 m-1} \delta^{2}\right)^{1 / m-1}-2 j n \geq 0,
$$

which is equivalent to

$$
c^{m}+2 j^{2 m-1} \delta^{2} \leq\left(\frac{2 n m}{j^{2 m-2}}\right)^{m /(1-m)}=\frac{j^{2 m}}{(2 n m)^{m /(m-1)}},
$$

and this happens if $j$ is large enough. Thus, for such $j, w_{c, j}$ is superparabolic, by Theorem 3.5. It now follows that $\left\{w_{c, j}\right\}$ (after renumbering) is an upper barrier family, and thus $\xi_{0}$ is upper regular.

For lower regularity, let again $c \in \mathbf{Q}_{+}$be arbitrary. This time let

$$
v_{c, j}=\left(c^{m}-j|x|^{2}-j a t\right)_{+}^{1 / m} \text { and } \Theta_{c, j}=\left\{(x, t) \in \Theta: v_{c, j}(x, t)>0\right\} \text {, }
$$

where $a=2 n m c^{m-1}$. In $\Theta_{c, j}$ we have

$$
\partial_{t} v_{c, j}=-\frac{j a}{m}\left(c^{m}-j|x|^{2}-j a t\right)^{1 / m-1} \leq-\frac{j a}{m} c^{1-m}=-2 j n
$$


and $\Delta v_{c, j}^{m}=-j \Delta|x|^{2}=-2 j n$. Hence $\partial_{t} v_{c, j}-\Delta v_{c, j}^{m} \leq 0$ and $v_{c, j}$ is subparabolic in $\Theta_{c, j}$, by Theorem 3.5. Lemma 5.8 shows that $v_{c, j}$ is subparabolic in $\Theta$. Hence, it follows that $\left\{v_{c, j}\right\}$ is a lower barrier family for positive boundary data and thus by Theorem $8.3, \xi_{0}=(0,0)$ is lower regular for positive boundary data.

Remark 9.2. Using the family $\left\{v_{c, j}\right\}$ above one can show that $\xi_{0}=(0,0)$ is lower regular for nonnegative data, in a similar way as the proof of $(2) \Rightarrow(1)$ in Theorem 8.3. We do not aim at developing the general theory of lower regular points for nonnegative data here.

\section{Independence of the Future}

The next result shows that regularity is independent of the future.

Theorem 10.1. Assume $m \geq 1$ and $\xi_{0}=(0,0) \in \partial \Theta$. Then $\xi_{0}$ is upper regular (lower regular for positive boundary data) with respect to $\Theta$ if and only if either $\xi_{0} \notin \partial \Theta_{-}$or $\xi_{0}$ is upper regular (lower regular for positive boundary data) with respect to $\Theta_{-}$.

Proof. We consider first the upper regularity.

If $\xi_{0}$ is upper regular with respect to $\Theta$, then either $\xi_{0} \notin \partial \Theta_{-}$or $\xi_{0}$ is upper regular with respect to $\Theta_{-}$, by Corollary 7.3.

As for the converse, if $\xi_{0} \notin \partial \Theta_{-}$, then $\xi_{0}$ is regular by Proposition 9.1. Thus it remains to consider the case when $\xi_{0}$ is upper regular with respect to $\Theta_{-}$.

Given $c \in \mathbf{Q}_{+}$and $j=1,2, \ldots$, we define for $(x, t) \in \Theta$ the function

$$
\psi_{c, j}=\left(c^{m}+j|x|^{2}+j b t^{2}\right)^{1 / m}
$$

with $b=m c^{m-1} / \operatorname{diam} \Theta$. By the proof of Theorem 7.2 we know that $\psi_{c, j}$ is subparabolic in $\Theta$.

Let $w_{c, j}=\underline{P}_{\Theta} \psi_{c, j}$. We want to show that $\left\{w_{c, j}\right\}$ is an upper barrier family at $\xi_{0}$ with respect to $\Theta$.

As $\psi_{c, j}$ is subparabolic and continuous, $\bar{S}_{\Theta} \psi_{c, j} \geq w_{c, j} \geq \psi_{c, j}$ in $\Theta$. By the upper regularity of $\xi_{0}$ with respect to $\Theta_{-}$as well as by Corollary 6.5 and Proposition 6.9(a), we see that

$$
\limsup _{\Theta_{-} \ni \zeta \rightarrow \xi_{0}} w_{c, j}(\zeta) \leq \limsup _{\Theta_{-} \ni \zeta \rightarrow \xi_{0}} \bar{S}_{\Theta} \psi_{c, j}(\zeta)=\limsup _{\Theta-\ni \zeta \rightarrow \xi_{0}} \bar{S}_{\Theta_{-}} \psi_{c, j}(\zeta) \leq \psi_{c, j}\left(\xi_{0}\right)
$$

Since $w_{c, j} \geq \psi_{c, j}$, we obtain

$$
\lim _{\Theta_{-} \ni \zeta \rightarrow \xi_{0}} w_{c, j}(\zeta)=\psi_{c, j}\left(\xi_{0}\right)
$$

Moreover, by the continuity of $w_{c, j}$ in $\Theta$ we have

$$
\lim _{\Theta \backslash \Theta_{+} \ni \zeta \rightarrow \xi_{0}} w_{c, j}(\zeta)=\psi_{c, j}\left(\xi_{0}\right) .
$$


Next, let

$$
\varphi= \begin{cases}\psi_{c, j} & \text { in } \partial \Theta \cap \partial \Theta_{+}, \\ w_{c, j} & \text { in } \Theta \cap \partial \Theta_{+} .\end{cases}
$$

Then $\varphi$ is continuous at $\xi_{0}$, by (10.1).

If $u \in \mathscr{L}_{\psi_{c, j}}(\Theta)$, then $\left.u\right|_{\Theta_{+}} \in \mathscr{L}_{\varphi}\left(\Theta_{+}\right)$, by the definitions of $\varphi$ and $w_{c, j}$. Hence

$$
w_{c, j}=\underline{P}_{\Theta} \psi_{c, j} \leq \underline{P}_{\Theta_{+}} \varphi \text { in } \Theta_{+} .
$$

If $v \in \widetilde{\mathscr{L}}_{\varphi}\left(\Theta_{+}\right)$then, since $w_{c, j} \geq \psi_{c, j}$ in $\Theta$, we have

$$
\limsup _{\Theta_{+} \ni \zeta \rightarrow \xi} v(\zeta)<\varphi(\xi) \leq \liminf _{\Theta_{+} \ni \zeta \rightarrow \xi} w_{c, j}(\zeta) \text { for all } \xi \in \partial \Theta_{+} .
$$

Thus, by Theorem 5.3, $v \leq w_{c, j}$ in $\Theta_{+}$. Taking supremum over all $v \in \widetilde{\mathscr{L}}_{\varphi}\left(\Theta_{+}\right)$ shows that

$$
\underline{S}_{\Theta_{+}} \varphi \leq w_{c, j} \leq \underline{P}_{\Theta_{+}} \varphi \text { in } \Theta_{+} .
$$

By Proposition 9.1, the initial points are always regular. Thus using also Proposition $6.9(\mathrm{~d})$, we see that

$$
\lim _{\Theta_{+} \ni \zeta \rightarrow \xi_{0}} \underline{S}_{\Theta_{+}} \varphi(\zeta)=\lim _{\Theta_{+} \ni \zeta \rightarrow \xi_{0}} \underline{P}_{\Theta_{+}} \varphi(\zeta)=\psi_{c, j}\left(\xi_{0}\right)
$$

Therefore, we obtain

$$
\lim _{\Theta_{+} \ni \zeta \rightarrow \xi_{0}} w_{c, j}(\zeta)=\varphi\left(\xi_{0}\right)
$$

which, together with (10.1), shows that

$$
\lim _{\Theta \ni \zeta \rightarrow \xi_{0}} w_{c, j}(\zeta)=\psi_{c, j}\left(\xi_{0}\right)
$$

All this together now allows us to conclude that $\left\{w_{c, j}\right\}$ is indeed an upper barrier family at $\xi_{0}$ with respect to $\Theta$. Thus, by Theorem 7.2, $\xi_{0}$ is upper regular with respect to $\Theta$.

Finally, we turn to the lower regularity (for positive boundary data). The proof is similar to the upper regularity case above, and the first part is analogous. For the main part, we let $0<\alpha<\gamma<1 / m, d=2+\log \operatorname{diam} \Theta$ and define

$$
v_{c, j}(x, t)=\left\{\begin{array}{ll}
c^{-1 / \gamma}+j^{\alpha} t^{2}+\frac{j}{d-\log |x|}, & x \neq 0, \\
c^{-1 / \gamma}+j^{\alpha} t^{2}, & x=0,
\end{array} \quad \text { and } \quad \psi_{c, j}=v_{c, j}^{-\gamma} .\right.
$$

This time $\psi_{c, j}$ is superparabolic if $j \geq j_{0}(c)$, by the proof of Theorem 8.3. The rest of the proof is the same as for upper regularity, with the direction reversed, and using Proposition 6.9(c) and Theorem 8.3 at appropriate places. We omit the details. 


\section{Regularity of Cylinders}

In this section, we will show that the boundary regularity for the boundary value problem (2.4) for the porous medium equation in a cylinder is determined by the elliptic regularity of the corresponding spatial set. For this reason, we recall the concept of capacity and the elliptic Wiener criterion.

For a bounded set $E \subset \mathbf{R}^{n}$, we define the capacity of $E$ as

$$
\operatorname{cap}(E)=\inf _{u} \int_{\mathbf{R}^{n}}\left(|\nabla u|^{2}+|u|^{2}\right) d x,
$$

where the infimum is taken over all $u \in C_{0}^{\infty}\left(\mathbf{R}^{n}\right)$ such that $u \geq 1$ in a neighbourhood of $E$.

With this definition, the Wiener criterion [44] characterizes the regular boundary points of a bounded open set $U \subset \mathbf{R}^{n}$, i.e. those boundary points $x_{0} \in \partial U$ at which every solution of the elliptic boundary value problem

$$
\begin{cases}\Delta u=0 & \text { in } U, \\ u=g \in C(\partial U) & \text { on } \partial U,\end{cases}
$$

attains its continuous boundary values $g$. We call these points elliptic regular. More precisely, $x_{0} \in \partial U$ is elliptic regular if and only if the complement of $U$ is thick at $x_{0}$, i.e., if

$$
\int_{0}^{1} \frac{\operatorname{cap}\left(B\left(x_{0}, r\right) \backslash U\right)}{r^{n-2}} \frac{d r}{r}=\infty
$$

(see Wiener [44], Littman-Stampacchia-Weinberger [36, Theorem 9.2] or MalýZiemer [37, Theorem 4.24]).

It is well known, and rather straightforward, that the Wiener condition (11.2) holds e.g. if $x_{0}$ is not a point of density of $U$ or if $U$ satisfies the following porosity condition: there is $c>0$ and a sequence $r_{k} \rightarrow 0$ such that for every $k=1,2, \ldots$, the set $B\left(x_{0}, r_{k}\right) \backslash U$ contains a ball of radius $c r_{k}$. In particular, the famous cone and corkscrew conditions are sufficient for boundary regularity. Thus, our results in this section apply to a much larger class of (unions of) cylinders than the ones considered in Abdulla [1,2].

Theorem 11.1. Let $m \geq 1$ and $\Theta=U_{t_{1}, t_{2}}$. Also let $\xi_{0}=\left(x_{0}, t_{0}\right) \in \partial \Theta$, where $t_{1}<t_{0} \leq t_{2}$. Then $\xi_{0}$ is regular for positive boundary data if and only if $x_{0}$ is elliptic regular with respect to $U$.

Note that by the main result in Kinnunen-Lindqvist-Lukkari [30], continuous functions are resolutive for cylinders (see also Remark 6.3). Also, if $t_{0}=t_{1}$ then $\xi_{0}$, being an earliest point, is always regular for positive boundary data, by Proposition 9.1.

Proof. Without loss of generality we may assume that $\xi_{0}=(0,0)$, and thus that $t_{1}<0 \leq t_{2}$. 
Assume first that $x_{0}$ is elliptic regular with respect to $U$. Let $\varphi(x)=|x|, x \in \mathbf{R}^{n}$, and let $v$ be the unique classical solution of

$$
\left\{\begin{array}{l}
\Delta v=-1 \text { in } U \\
v-\varphi \in W_{0}^{1,2}(U)
\end{array}\right.
$$

which exists by Theorem 4.3 in Gilbarg-Trudinger [25]. Then $v$ is superharmonic in $U$. Since $\varphi$ is subharmonic (by a straightforward calculation), we have $v \geq \varphi$ a.e., by Lemma 3.18 in Heinonen-Kilpeläinen-Martio [27]. As $v$ and $\varphi$ are continuous, $v \geq \varphi$ everywhere in $U$. Since $x_{0}$ is elliptic regular, it satisfies the Wiener test in Wiener's criterion. Hence it follows from Gariepy-Ziemer [24, Theorem 2.2] (or Malý-Ziemer [37, Theorem 4.28]) that $\lim _{U \ni x \rightarrow 0} v(x)=\varphi(0)=0$.

We need to create barrier families both for upper and lower regularity. We begin with upper regularity and let $c \in \mathbf{Q}_{+}$be arbitrary. Let

$$
w_{c, j}=\left(c^{m}+j v+a j t^{2}\right)^{1 / m},
$$

where $a=c^{m-1} m / 2 \operatorname{diam} \Theta$. Then, as $1 / m-1<0$,

$$
\partial_{t} w_{c, j}=\frac{2 a j t}{m}\left(c^{m}+j v+a j t^{2}\right)^{1 / m-1} \geq-\frac{2 a j \operatorname{diam} \Theta}{m} c^{1-m},
$$

while $\Delta w_{c, j}^{m}=j \Delta v=-j$. Hence

$$
\partial_{t} w_{c, j}-\Delta w_{c, j}^{m} \geq j\left(1-\frac{2 a \operatorname{diam} \Theta}{m} c^{1-m}\right)=0,
$$

and thus $w_{c, j}$ is superparabolic in $\Theta$, by Theorem 3.5. As $\lim _{U \ni x \rightarrow 0} v(x)=0$ and $v \geq \varphi$, it follows that $\left\{w_{c, j}\right\}$ is an upper barrier family and therefore by Theorem 7.2, $\xi_{0}=(0,0)$ is upper regular.

Next, we turn to lower regularity for positive boundary data. Let again $c \in \mathbf{Q}_{+}$ be arbitrary. This time we let

$$
\begin{aligned}
& u_{c, j}=\max \left\{c^{m}-j v-b j^{1 / m} t^{2}, \frac{1}{j}\right\}^{1 / m}, \\
& \Theta_{c, j}=\left\{(x, t) \in \Theta: u_{c, j}(x, t)^{m}>\frac{1}{j}\right\},
\end{aligned}
$$

where $b=m / 2 \operatorname{diam} \Theta$ and $v$ is the same function as above. In $\Theta_{c, j}$, which is open as $v$ is continuous, we have

$$
\partial_{t} u_{c, j}=-\frac{2 b j^{1 / m} t}{m}\left(c^{m}-j v-b j^{1 / m} t^{2}\right)^{1 / m-1} \leq \frac{2 b j^{1 / m} \operatorname{diam} \Theta}{m} j^{1-1 / m}=j,
$$

while $\Delta u_{c, j}^{m}=-j \Delta v=j$. Hence

$$
\partial_{t} u_{c, j}-\Delta u_{c, j}^{m} \leq j-j=0,
$$

and thus $u_{c, j}$ is subparabolic in $\Theta_{c, j}$, by Theorem 3.5. As $v$ is continuous, $u_{c, j}$ is also continuous. Hence, Lemma 5.8 shows that $u_{c, j}$ is subparabolic in $\Theta$. Since 
$\lim _{U \ni x \rightarrow 0} v(x)=0$ and $v \geq \varphi$, it follows that $\left\{u_{c, j}\right\}$ is a lower barrier family for positive boundary data and thus by Theorem $8.3, \xi_{0}=(0,0)$ is lower regular for positive boundary data.

Assume now instead that $\xi_{0}=(0,0)$ is regular for positive boundary data. We let $\psi$ be a continuous function on $\partial U$ and let $h$ be the harmonic Perron solution with boundary values $\psi$ with respect to $U$. We need to show that $\lim _{U \ni x \rightarrow 0} h(x)=\psi(0)$. As we can scale and add constants to harmonic functions we may assume that $1 \leq \psi \leq 2$, and thus also $1 \leq h \leq 2$. Let

$$
f(x, t)= \begin{cases}\psi(x), & \text { if } x \in \partial U \text { and } t_{1}<t<t_{2}, \\ h(x), & \text { if } x \in \bar{U}, \text { and } t=t_{1} \text { or } t=t_{2} .\end{cases}
$$

By Theorem 10.1 we may assume that $t_{2}>0$. Then $1 \leq f \leq 2$ on $\partial \Theta$ and $f$ is continuous at $\xi_{0}$. Moreover, if $u$ belongs to the elliptic lower class for the harmonic Perron solution of $\psi$, then $\tilde{u}(x, t)=\max \{u(x), 1\} \in \mathscr{L}_{f}$ and hence $\underline{P} f(x, t) \geq h(x)$ for $(x, t) \in \Theta$. By Proposition 6.9(a),

$$
\limsup _{U \ni x \rightarrow 0} h(x) \leq \limsup _{U \ni x \rightarrow 0} \underline{P} f(x, 0) \leq \limsup _{U \ni x \rightarrow 0} \bar{S} f(x, 0) \leq f(0,0)=\psi(0) .
$$

It follows similarly that $\liminf _{U \ni x \rightarrow 0} h(x) \geq \psi(0)$, and thus $\lim _{U \ni x \rightarrow 0} h(x)=$ $\psi(0)$. Hence 0 is elliptic regular with respect to $U$.

A related result is proved by Ziemer in [45, Theorem 4.4]. He considers general degenerate parabolic equations, which include the porous medium equation with $m>1$ as a special case. He deals with signed weak solutions $u$ in a cylinder $U_{t_{1}, t_{2}}$, and assumes them to be bounded. The boundary data $f$ belong to the Sobolev space $W_{2}^{1,1}\left(\mathbf{R}^{n+1}\right)$ of functions which, together with their distributional first derivatives, belong to $L^{2}\left(\mathbf{R}^{n+1}\right)$, and $\left.f\right|_{\mathbf{R}^{n+1} \backslash U_{t_{1}, t_{2}}}$ is continuous. The boundary condition on $\partial U_{t_{1}, t_{2}}$ is taken in a weak (Sobolev) sense, that is $u-f$ is assumed to be in the $W_{2}^{1,1}\left(U_{t_{1}, t_{2}}\right)$-closure of smooth functions with compact support in $U_{t_{1}, t_{2}}$. If $\xi_{0}=\left(x_{0}, t_{0}\right) \in \partial U \times\left(t_{1}, t_{2}\right)$ and $x_{0}$ is elliptic regular, then by [45, Theorem 4.4],

$$
\lim _{U_{t_{1}, t_{2}} \ni \xi \rightarrow \xi_{0}} u(\xi)=f\left(\xi_{0}\right) .
$$

There is no restriction on the boundary behaviour of $f$ at $\xi_{0}$, which can be positive, negative, or vanish, but the condition for continuity is only proved to be sufficient.

Our aim in the rest of this section is to obtain the following generalization of Abdulla's unique solvability result (Theorem 2.4) for $m \geq 1$ and positive boundary data (note that it is a generalization also in the case when $\Theta$ is just one cylinder):

Theorem 11.2. Let $m \geq 1$ and $\Theta$ be a finite union of cylinders. Assume that the time-sections

$$
\Theta(T):=\{(x, t) \in \Theta: t=T\}
$$

are bounded elliptic regular open sets in $\mathbf{R}^{n}$ satisfying

$$
\Theta\left(T_{1}\right) \subset \Theta\left(T_{2}\right) \text { whenever } T_{\min }<T_{1}<T_{2}<T_{\max },
$$

where $T_{\min }:=\inf \{t:(x, t) \in \Theta\}$ and $T_{\max }:=\sup \{t:(x, t) \in \Theta\}$. Then 
(a) every point in the parabolic boundary $\partial_{p} \Theta$ is regular for positive boundary data;

(b) every positive $f \in C\left(\partial_{p} \Theta\right)$ is resolutive and

$$
u:=P f=\underline{S} f=\underline{P} f=\bar{P} f=\bar{S} f
$$

is the unique function in $C(\bar{\Theta})$ which is parabolic in $\Theta$ and takes the boundary values $u=f$ on the parabolic boundary $\partial_{p} \Theta$;

(c) every nonnegative $f \in C\left(\partial_{p} \Theta\right)$ is resolutive and for every $\xi_{0} \in \partial_{p} \Theta$, either

$$
\lim _{\Theta \ni \xi \rightarrow \xi_{0}} P f(\xi)=f\left(\xi_{0}\right) \quad \text { or } \quad 0=\liminf _{\Theta \ni \xi \rightarrow \xi_{0}} P f(\xi) \leq \limsup _{\Theta \ni \xi \rightarrow \xi_{0}} P f(\xi) \leq f\left(\xi_{0}\right) .
$$

The last part also generalizes the resolutivity result in Kinnunen-LindqvistLukkari [30, Theorem 5.1] (for general cylinders) to certain finite unions of cylinders.

We will divide the proof into several results. Since they have independent interest we formulate them in greater generality than Theorem 11.2.

Theorem 11.3. Let $m \geq 1$ and $\Theta$ be a finite union of cylinders. Let $h \in C(\partial \Theta)$ be nonnegative. Then there is at most one $u \in C(\bar{\Theta})$ that is parabolic in $\Theta$ and takes the boundary values $u=h$ on the parabolic boundary $\partial_{p} \Theta$.

Proof. Let $u$ and $v$ be two solutions of the boundary value problem under consideration. Theorem 5.4 shows that $v \leq u \leq v$ in $\Theta$ and hence, by continuity, in $\bar{\Theta}$.

Proposition 11.4. Let $m>0$. For $T \in \mathbf{R}$, let

$$
\partial_{T} \Theta=\{\xi=(x, t) \in \partial \Theta: t<T\}
$$

and assume that $f: \partial \Theta_{T} \rightarrow[0, \infty)$ is bounded on $\partial \Theta_{T}$ and continuous on $\partial_{T} \Theta$. Then the following are true:

(a) If every $\xi \in \partial_{T} \Theta$ is upper regular, then

$$
\bar{P}_{\Theta_{T}} f \leq \bar{S}_{\Theta_{T}} f=\underline{P}_{\Theta_{T}} f .
$$

(b) If every $\xi \in \partial_{T} \Theta$ is lower regular for positive boundary data and $f$ is bounded away from 0 , then

$$
\underline{P}_{\Theta_{T}} f \geq \underline{S}_{\Theta_{T}} f=\bar{P}_{\Theta_{T}} f .
$$

(c) If every $\xi \in \partial_{T} \Theta$ is lower regular for nonnegative boundary data, then

$$
\bar{P}_{\Theta_{T}} f \leq \underline{P}_{\Theta_{T}} f
$$

(d) If the comparison principle $\underline{P}_{\Theta_{T}} f \leq \bar{P}_{\Theta_{T}} f$ is valid on $\Theta_{T}$ then in any of the above cases (a)-(c), $f$ is resolutive in $\Theta_{T}$. 
(e) Let $0<h \in C\left(\partial \Theta_{T}\right)$ be positive. Then in any of the above cases (a)-(c), there is a countable set $A \subset(0,1)$ such that

$$
\underline{P}_{\Theta_{T}}(f+a h)=\bar{P}_{\Theta_{T}}(f+a h) \text { for every } a \in(0,1) \backslash A,
$$

i.e. $f+$ ah is resolutive in $\Theta_{T}$.

This result naturally combines with Lemma 6.4 and Corollary 6.5. In particular, it can be applied to bounded continuous functions defined only on $\partial_{T} \Theta$ and extended arbitrarily in a bounded way to $\partial \Theta_{T}$ and $\partial \Theta$.

Proof. (a) Proposition 6.9 (a), together with the upper regularity assumption, shows that

$$
\limsup _{\Theta \ni \zeta \rightarrow \xi} \bar{S}_{\Theta_{T}} f(\zeta) \leq f(\xi) \text { for every } \xi \in \partial_{T} \Theta
$$

Since $\bar{S}_{\Theta_{T}} f$ is parabolic in $\Theta_{T}$, this together with Lemma 6.4 (applied to $\Theta_{T}$ ) shows that

$$
\bar{S}_{\Theta_{T}} f \leq \underline{P}^{T} f=\underline{P}_{\Theta_{T}} f .
$$

The converse inequality $\underline{P}_{\Theta_{T}} f \leq \bar{S}_{\Theta_{T}} f$ follows from Theorem 5.3, while Remark 6.3 shows that $\bar{P}_{\Theta_{T}} f \leq \bar{S}_{\Theta_{T}} f$.

(b) This is shown in the same way as (a), by interchanging the role of the upper and lower solutions, and taking into account the positivity of $f$ and Proposition 6.9 (c).

(c) As in (a), Proposition 6.9 (b), together with the lower regularity assumption, implies that

$$
\limsup _{\Theta \ni \eta \rightarrow \xi} \underline{P}_{\Theta_{T}} f(\eta) \geq f(\xi) \text { for every } \xi \in \partial_{T} \Theta
$$

and hence, by Lemma 6.4 (applied to $\Theta_{T}$ ), $\underline{P}_{\Theta_{T}} f \geq \bar{P}^{T} f=\bar{P}_{\Theta_{T}} f$.

(d) This is a direct consequence of (a)-(c) and the general inequalities

$$
\underline{S}_{\Theta_{T}} f \leq \underline{P}_{\Theta_{T}} f \leq \bar{S}_{\Theta_{T}} f \quad \text { and } \quad \underline{S}_{\Theta_{T}} f \leq \bar{P}_{\Theta_{T}} f \leq \bar{S}_{\Theta_{T}} f .
$$

(e) Let $E$ be a countable dense subset of $\Theta_{T}$. For each $\xi \in E$ the function $(0,1) \ni a \mapsto \underline{P}(f+a h)(\xi)$ is nondecreasing and thus has jumps for at most countably many values of $a$; let $A_{\xi}$ be the set of these values of $a$. Then $A:=$ $\bigcup_{\xi \in E} A_{\xi}$ is also countable.

Now let $a \in(0,1) \backslash A$. Then, for every $\xi \in E$, using the positivity of $h$ on $\partial \Theta_{T}$ and the elliptic comparison principle (Theorem 5.3), together with (a), (b) or (c) above, we see that

$$
\underline{P}(f+a h)(\xi)=\lim _{b \rightarrow a^{-}} \underline{P}(f+b h)(\xi) \leq \bar{P}(f+a h)(\xi) \leq \underline{P}(f+a h)(\xi) .
$$

Thus $\underline{P}(f+a h)=\bar{P}(f+a h)$ in $E$ and as they both are continuous this holds everywhere in $\Theta_{T}$, i.e. $f+a h$ is resolutive. 
Theorem 11.5. Let $m \geq 1$ and let $f: \partial \Theta \rightarrow[0, \infty)$ be bounded. Assume in addition that $f$ is continuous at $\xi_{0} \in \partial \Theta, f\left(\xi_{0}\right)>0$ and that $\xi_{0}$ is lower regular for positive boundary data. Then either

$$
\liminf _{\Theta \ni \xi \rightarrow \xi_{0}} \bar{P} f(\xi) \geq f\left(\xi_{0}\right) \text { or } \liminf _{\Theta \ni \xi \rightarrow \xi_{0}} \bar{P} f(\xi)=0 .
$$

Similarly, either $\liminf _{\Theta \ni \xi \rightarrow \xi_{0}} \bar{S} f(\xi) \geq f\left(\xi_{0}\right)$ or $\liminf _{\Theta \ni \xi \rightarrow \xi_{0}} \bar{S} f(\xi)=0$.

Proof. Assume that $\liminf _{\Theta \ni \xi \rightarrow \xi_{0}} \bar{P} f(\xi)>0$. Then there exists $\varepsilon>0$ and a ball $B \ni \xi_{0}$, such that $\bar{P} f \geq \varepsilon$ on $\Theta \cap \bar{B}$ and $f \geq \varepsilon$ on $\partial \Theta \cap \bar{B}$. Thus, the function

$$
h= \begin{cases}f & \text { on } \partial \Theta \cap \bar{B}, \\ \bar{P} f & \text { on } \partial B \cap \Theta,\end{cases}
$$

is bounded away from zero on $\partial(B \cap \Theta)$ and continuous at $\xi_{0}$.

Now, let $u \in \mathscr{U}_{f}(\Theta)$ be arbitrary. Then by definition,

$$
\liminf _{\Theta \ni \zeta \rightarrow \xi} u(\zeta) \geq f(\xi)=h(\xi) \text { for } \xi \in \partial \Theta \cap \bar{B}
$$

while the lower semicontinuity of $u$, together with the definition of $\bar{P} f$ and $h$, implies that

$$
\liminf _{\Theta \ni \zeta \rightarrow \xi} u(\zeta) \geq u(\xi) \geq \bar{P} f(\xi)=h(\xi) \text { for } \xi \in \partial B \cap \Theta .
$$

It follows that $u \in \mathscr{U}_{h}(\Theta \cap B)$ and taking infimum over all such $u$ yields

$$
\bar{P} f \geq \bar{P}_{B \cap \Theta} h \geq \underline{S}_{B \cap \Theta} h \text { in } B \cap \Theta .
$$

Corollary 8.4 shows that $\xi_{0}$ is lower regular for positive boundary data with respect to $B \cap \Theta$. We therefore conclude from Proposition 6.9(c) that

$$
\liminf _{\Theta \ni \zeta \rightarrow \xi_{0}} \bar{P} f(\zeta) \geq \liminf _{\Theta \ni \zeta \rightarrow \xi_{0}} \underline{S}_{B \cap \Theta} h(\zeta) \geq h\left(\xi_{0}\right)=f\left(\xi_{0}\right) .
$$

The case with $\bar{S} f$ is obtained similarly.

Finally, we are ready to prove Theorem 11.2.

Proof of Theorem 11.2. (a) There are two types of points in $\partial_{p} \Theta$ : those belonging to the lateral surface of one of the cylinders constituting $\Theta$ and those belonging to the flat bottom of one of these cylinders. Because of the assumption $\Theta\left(T_{1}\right) \subset \Theta\left(T_{2}\right)$, none of the parabolic boundary points belongs to the top of any of the cylinders. (Here by the top of $U_{t_{1}, t_{2}}$ we mean $\partial U_{t_{1}, t_{2}} \backslash \partial_{p} U_{t_{1}, t_{2}}$.)

The lateral points are regular for positive boundary data by Theorem 11.1, together with Propositions 7.4 and 8.5, while the bottom points are regular for positive boundary data by Proposition 9.1 .

(b) The resolutivity and the identities in (11.3) follow from Proposition 11.4 (a), (b) and (d), together with the parabolic comparison principle, Theorem 5.4. The uniqueness is a direct consequence of Theorem 11.3. 
The continuity of $u$ on $\partial_{p} \Theta$ follows from Proposition 6.9(d), while Theorem 5.16.1 in DiBenedetto-Gianazza-Vespri [23] shows that $u$ has a continuous extension to all points on the top level $\partial \Theta \backslash \partial_{p} \Theta$.

(c) As in (b), the resolutivity follows from Proposition 11.4 (a) and (d), together with Theorem 5.4. Proposition 6.9 (a) then shows that

$$
\limsup _{\Theta \ni \xi \rightarrow \xi_{0}} P f(\xi) \leq f\left(\xi_{0}\right) \text { for all } \xi_{0} \in \partial_{p} \Theta
$$

while Theorem 11.5 implies that (11.4) holds.

Acknowledgements. This research started while the authors were visiting Institut MittagLeffler in 2013, we thank the institute for the kind hospitality.

Conflict of interest: The authors declare that they have no conflict of interest.

Funding: A. B. and J. B. were supported by the Swedish Research Council, Grant Nos. 621-2011-3139, 621-2014-3974 and 2016-03424. J. S. was supported by the Academy of Finland grant 259363 and a Väisälä foundation travel grant.

Open Access This article is distributed under the terms of the Creative Commons Attribution 4.0 International License (http://creativecommons.org/licenses/by/4.0/), which permits unrestricted use, distribution, and reproduction in any medium, provided you give appropriate credit to the original author(s) and the source, provide a link to the Creative Commons license, and indicate if changes were made.

\section{Appendix A. Proof of Theorem 3.4}

Kuusi [32], working with the $p$-parabolic equation (1.2), observed that the crucial step towards establishing inner regularity results like Theorem 3.4 is a supremum estimate for subsolutions. Such results had earlier been obtained using weak Harnack inequalities, which are more difficult to deduce than supremum estimates. Avelin-Lukkari [7] later adapted Kuusi's argument to supersolutions of the porous medium equation (establishing Theorem 3.3).

First, we set the notation

$$
Q\left(x_{0}, t_{0}, \rho\right)=B\left(x_{0}, \rho\right) \times\left(t_{0}-\rho^{2}, t_{0}+\rho^{2}\right) \text { and } Q(\rho)=Q(0,0, \rho) .
$$

Proposition A.1. (Supremum estimate for subsolutions) Let $m \geq 1$, $u$ be a bounded nonnegative subsolution in $Q\left(x_{0}, t_{0}, \rho\right), 0<\sigma<1$ and $M \geq 0$. Then

$$
\operatorname{ess}_{Q\left(x_{0}, t_{0}, \sigma \rho\right)}(u-M) \leq C\left(\frac{1}{|Q(\rho)|} \iint_{Q\left(x_{0}, t_{0}, \rho\right)}(u-M)_{+}^{2} d x d t\right)^{1 / \lambda},
$$

where $\lambda=2+\frac{(m-1) n}{2}$, and $C$ only depends on $n, m, \sigma$ and $L:=\operatorname{ess}_{\sup } Q\left(x_{0}, t_{0}, \rho\right)$, but not on $u, M$ and $\rho$.

To prove this estimate we modify the technique used by Andreucci [4]. Note that we do not have the extra corrective term that appears in [4], since here we directly assume that the height of the cylinder is $\rho^{2}$ and allow $C$ to depend on $L$, which is sufficient for our purposes.

Before proving Proposition A.1, we show how this estimate is used to obtain Theorem 3.4. 
Proof of Theorem 3.4. Let $\left(x_{0}, t_{0}\right) \in \Theta$ be a Lebesgue point of $u$. Without loss of generality we assume that $\left(x_{0}, t_{0}\right)=(0,0)$. We want to show that $u^{*}(0,0)=u(0,0)$. First of all,

$$
u^{*}(0,0) \geq \lim _{\rho \rightarrow 0} \frac{1}{|Q(\rho)|} \iint_{Q(\rho)} u d x d t=u(0,0) .
$$

For the converse inequality, first choose $r$ such that $Q(r) \Subset \Theta$. Let $L=\operatorname{ess}_{\sup } Q(r)$, which is finite, see e.g. Andreucci [4]. Then, by Proposition A.1 (with $M=u(0,0)$ and $\sigma=\frac{1}{2}$ ), we have for any $0<\rho<r$,

$$
\begin{aligned}
\underset{Q(\rho / 2)}{\operatorname{ess} \sup (u-u(0,0))} & \leq C\left(\frac{1}{|Q(\rho)|} \iint_{Q(\rho)}(u-u(0,0))_{+}^{2} d x d t\right)^{1 / \lambda} \\
& \leq C L^{1 / \lambda}\left(\frac{1}{|Q(\rho)|} \iint_{Q(\rho)}|u-u(0,0)| d x d t\right)^{1 / \lambda},
\end{aligned}
$$

which tends to 0 , as $\rho \rightarrow 0$, since $(0,0)$ is a Lebesgue point. Thus we conclude that $u^{*}(0,0) \leq u(0,0)$.

Now we turn to the proof of Proposition A.1.

Proof of Proposition A.1. Without loss of generality we assume that $\left(x_{0}, t_{0}\right)=(0,0)$. Define for all $j \geq 0$,

$$
\begin{array}{ll}
t_{j}^{ \pm}= \pm\left(\sigma^{2} \rho^{2}+\frac{1-\sigma^{2}}{2^{j}} \rho^{2}\right), & \rho_{j}=\sigma \rho+\frac{1-\sigma}{2^{j}} \rho, \\
B_{j}=B\left(0, \rho_{j}\right), & Q_{j}=B_{j} \times\left(t_{j}^{-}, t_{j}^{+}\right) .
\end{array}
$$

Note that

$$
Q(\sigma \rho) \Subset Q_{j+1} \Subset Q_{j} \Subset Q_{0}=Q(\rho) .
$$

We also consider $C^{\infty}$-cutoff functions $\zeta_{j}$ such that $0 \leq \zeta_{j} \leq 1$ and

$$
\begin{aligned}
\zeta_{j} & \equiv 0 \text { outside } Q_{j}, \\
\left|\nabla \zeta_{j}\right| & \leq \frac{2^{j+2}}{(1-\sigma) \rho}, \quad 0 \leq\left|\partial_{t} \zeta_{j}\right| \leq \frac{2^{j+2}}{\left(1-\sigma^{2}\right) \rho^{2}} .
\end{aligned}
$$

Fix $k>0$ to be chosen later, set

$$
u_{j}=\left(u-M-k_{j+1}\right)_{+}, \quad \text { where } k_{j}=k-\frac{k}{2^{j+1}},
$$

and use $f_{j}=2 u_{j} \zeta_{j}^{2}$ as a test function. Note that

$$
\partial_{t} u_{j}=\partial_{t} u \text { and } \nabla u_{j}=\nabla u
$$

a.e. in the set where $u_{j} \neq 0$ (and that $\partial_{t} u_{j}=\nabla u_{j}=0$ a.e. otherwise). Some of the calculations below are formal. As far as the time derivative $\partial_{t} u_{j}$ is concerned, the calculations can be made rigorous by means of a Steklov averaging process, cf. e.g. DiBenedetto-GianazzaVespri [23, pp. 21 and 35], or by a mollification in time, see e.g. Kinnunen-Lindqvist [29, pp. 141-143]. Another difficulty is represented by $\nabla u$, since in general only $\nabla u^{m}$ is welldefined: for a way to deal with this second issue, see e.g. Bögelein-Duzaar-Gianazza [12, Lemma 2.2]. Note, however, that in the integrals below, $\nabla u$ is only considered at points, where $u>k_{j}$, and thus $\nabla u=m^{-1}\left(u^{m}\right)^{1 / m-1} \nabla u^{m}$ is well defined. 
For all $\tau \in\left(t_{j}^{-}, t_{j}^{+}\right)$, the time part of the weak formulation of subsolutions, within $Q_{j}^{\tau}:=$ $B_{j} \times\left(t_{j}^{-}, \tau\right)$, becomes

$$
\begin{aligned}
\iint_{Q_{j}^{\tau}} f_{j} \partial_{t} u d x d t & =\iint_{Q_{j}^{\tau}} \zeta_{j}^{2} \partial_{t} u_{j}^{2} d x d t \\
& \geq \int_{B_{j}} u_{j}(x, \tau)^{2} \zeta_{j}(x, \tau)^{2} d x-\frac{2^{j+3}}{\left(1-\sigma^{2}\right) \rho^{2}} \iint_{Q_{j}^{\tau}} u_{j}^{2} d x d t
\end{aligned}
$$

where we used partial integration with respect to $d t$ and properties (A.2) of the cutoff function $\zeta_{j}$ in the second step.

Now we turn to the elliptic part. We have

$$
\nabla f_{j}=2 \zeta_{j}^{2} \nabla u_{j}+4 u_{j} \zeta_{j} \nabla \zeta_{j} \text { a.e. }
$$

and hence, using (A.3), we see that

$$
\begin{aligned}
\iint_{Q_{j}^{\tau}} \nabla u^{m} \cdot \nabla f_{j} d x d t \geq & 2 m \iint_{Q_{j}^{\tau}} u^{m-1} \zeta_{j}^{2}\left|\nabla u_{j}\right|^{2} d x d t \\
& -4 m \iint_{Q_{j}^{\tau}} u^{m-1}\left(\zeta_{j}\left|\nabla u_{j}\right|\right)\left(u_{j}\left|\nabla \zeta_{j}\right|\right) d x d t .
\end{aligned}
$$

Next, using Young's inequality, we get that for every $\theta>0$,

$$
\begin{aligned}
\iint_{Q_{j}^{\tau}} \nabla u^{m} \cdot \nabla f_{j} d x d t \geq & (2 m-2 m \theta) \iint_{Q_{j}^{\tau}} u^{m-1} \zeta_{j}^{2}\left|\nabla u_{j}\right|^{2} d x d t \\
& -\frac{2 m}{\theta} \iint_{Q_{j}^{\tau}} u^{m-1} u_{j}^{2}\left|\nabla \zeta_{j}\right|^{2} d x d t
\end{aligned}
$$

We now make the choice $\theta=\frac{1}{2}$. Using also that $L \geq u \geq k_{j+1} \geq k / 2$ when $u_{j} \neq 0$ (and that $\nabla u_{j}=0$ a.e. otherwise), together with (A.2), we conclude that

$$
\begin{aligned}
\iint_{Q_{j}^{\tau}} \nabla u^{m} \cdot \nabla f_{j} d x d t \geq & \frac{m k^{m-1}}{2^{m-1}} \iint_{Q_{j}^{\tau}} \zeta_{j}^{2}\left|\nabla u_{j}\right|^{2} d x d t \\
& -\frac{4^{j+3} m L^{m-1}}{(1-\sigma)^{2} \rho^{2}} \iint_{Q_{j}^{\tau}} u_{j}^{2} d x d t .
\end{aligned}
$$

Combining this with (A.4) and using that $u$ is a subsolution yields the energy estimate

$$
\int_{B_{j}} u_{j}(x, \tau)^{2} \zeta_{j}(x, \tau)^{2} d x+k^{m-1} \iint_{Q_{j}^{\tau}} \zeta_{j}^{2}\left|\nabla u_{j}\right|^{2} d x d t \leq \frac{4^{j} \widehat{\gamma}}{2 \rho^{2}} \iint_{Q_{j}^{\tau}} u_{j}^{2} d x d t,
$$

where $\widehat{\gamma}$ only depends on $m, \sigma$ and $L$, but not on $u, M, k, \rho$ or $j$. Taking the supremum separately of each term on the left-hand side and adding the estimates yields

$$
\begin{aligned}
& \sup _{t_{j}^{-}} \leq t \leq t_{j}^{+} \\
& \quad \leq \frac{4^{j} \widehat{\gamma}}{\rho^{2}} \iint_{Q_{j}} u_{j}(x, t)^{2} \zeta_{j}^{2}(x, t) d x+k^{m-1} \iint_{Q_{j}} \zeta_{j}^{2}\left|\nabla u_{j}\right|^{2} d x d t \\
& \quad \frac{4^{j} \widehat{\gamma}}{\rho^{2}} Y_{j},
\end{aligned}
$$


where

$$
Y_{j}:=\iint_{Q_{j}}\left(u(x, t)-M-k_{j}\right)_{+}^{2} d x d t
$$

Next, we need an estimate for the measure of

$$
A_{j}=\left\{(x, t) \in Q_{j}: u_{j}(x, t)>0\right\}=\left\{(x, t) \in Q_{j}: u(x, t)>M+k_{j+1}\right\} .
$$

Using that $k_{j+1}-k_{j}=2^{-(j+2)} k$, we see that

$$
Y_{j} \geq \iint_{A_{j}}\left(u(x, t)-M-k_{j}\right)_{+}^{2} d x d t \geq 4^{-(j+2)} k^{2}\left|A_{j}\right| .
$$

Let $\alpha=2 /(n+2)$ and note that $1-\alpha=n /(n+2)$. By Hölder's inequality and the parabolic Sobolev embedding (see e.g. DiBenedetto-Gianazza-Vespri [23, Proposition 4.1, Chapter 1]), we obtain that

$$
\begin{aligned}
Y_{j+1} \leq & \iint_{Q_{j}}\left(u_{j} \zeta_{j}\right)^{2} d x d t \\
\leq & \left|A_{j}\right|^{\alpha}\left(\iint_{Q_{j}}\left(u_{j} \zeta_{j}\right)^{2 /(1-\alpha)} d x d t\right)^{1-\alpha} \\
\leq & \gamma(n)\left|A_{j}\right|^{\alpha}\left(\iint_{Q_{j}}\left(\left|\zeta_{j} \nabla u_{j}\right|^{2}+\left|u_{j} \nabla \zeta_{j}\right|^{2}\right) d x d t\right)^{1-\alpha} \\
& \times\left(\sup _{t_{j}^{-} \leq t \leq t_{j}^{+}} \int_{B_{j}} u_{j}(x, t)^{2} \zeta_{j}(x, t)^{2} d x d t\right)^{\alpha} .
\end{aligned}
$$

Estimating the factors on the right-hand side using (A.6) and (A.5) we can conclude that

$$
Y_{j+1} \leq A b^{j} Y_{j}^{1+\alpha}
$$

where

$$
b:=4^{1+\alpha} \text { and } A:=\frac{\widetilde{\gamma}\left(1+k^{1-m}\right)^{1-\alpha}}{k^{2 \alpha} \rho^{2}},
$$

and $\tilde{\gamma}$ only depends on $n, m, \sigma$ and $L$, but not on $u, M, k, \rho$ or $j$. It is now easily shown by induction (cf. DiBenedetto-Gianazza-Vespri [23, Lemma 5.1, Chapter 1]) that

$$
Y_{j} \leq A^{-1 / \alpha} b^{-1 / \alpha^{2}} b^{-j / \alpha} \rightarrow 0 \text { as } j \rightarrow \infty \text {, i.e. } u \leq M+k \text { in } Q(\sigma \rho),
$$

provided that $k$ is chosen so that $Y_{0} \leq A^{-1 / \alpha} b^{-1 / \alpha^{2}}$. Such a condition is satisfied if

$$
Y_{0} \leq \iint_{Q_{0}}(u(x, t)-M)_{+}^{2} d x d t \leq \tilde{\gamma}^{-1 / \alpha} b^{-1 / \alpha^{2}} \frac{k^{2} \rho^{n+2}}{\left(1+k^{1-m}\right)^{n / 2}} .
$$

Choosing

$$
k=\gamma^{\prime}\left(\left(\frac{Y_{0}}{\rho^{n+2}}\right)^{1 / \lambda}+\left(\frac{Y_{0}}{\rho^{n+2}}\right)^{1 / 2}\right),
$$

with $\gamma^{\prime}$ large enough only depending on $n, m, \sigma$ and $L$, will do. Since $Y_{0} / \rho^{n+2} \leq$ $2|B(0,1)| L^{2}$ and $1 / 2-1 / \lambda>0$, we see that

$$
\underset{Q(\sigma \rho)}{\operatorname{ess} \sup }(u-M) \leq k \leq \gamma\left(\frac{Y_{0}}{\rho^{n+2}}\right)^{1 / \lambda},
$$

where $\gamma$ again only depends on $n, m, \sigma$ and $L$. From this (A.1) follows. 


\section{References}

1. Abdulla, U.G.: On the Dirichlet problem for the nonlinear diffusion equation in nonsmooth domains. J. Math. Anal. Appl. 260, 384-403 (2001)

2. Abdulla, U.G.: Well-posedness of the Dirichlet problem for the non-linear diffusion equation in non-smooth domains. Trans. Am. Math. Soc. 357, 247-265 (2005)

3. Alt, H.W., Luckhaus, S.: Quasilinear elliptic-parabolic differential equations. Math. Z. 183, 311-341 (1983)

4. ANDREUCCI, D.: $L_{\text {loc }}^{\infty}$-estimates for local solutions of degenerate parabolic equations. SIAM J. Math. Anal. 22, 138-145 (1991)

5. Aronson, D.G., Crandall, M.G., Peletier, L.A.: Stabilization of solutions of a degenerate nonlinear diffusion problem. Nonlinear Anal. 6, 1001-1022 (1982)

6. Aronson, D.G., Peletier, L.A.: Large time behaviour of solutions of the porous medium equation in bounded domains. J. Differ. Equ. 39, 378-412 (1981)

7. Avelin, B., LukKari, T.: Lower semicontinuity of weak super-solutions to the porous medium equation. Proc. Am. Math. Soc. 143, 3475-3486 (2015)

8. Bauer, H.: Axiomatische Behandlung des Dirichletschen Problems für elliptische und parabolische Differentialgleichungen. Math. Ann. 146, 1-59 (1962)

9. BJÖRn, A., BJÖRn, J., GiAnAZZA, U.: The Petrovskiŭ criterion and barriers for degenerate and singular $p$-parabolic equations. Math. Ann. 368, 885-904 (2017)

10. BJörn, A., BJörn, J., Gianazza, U., PARviainen, M.: Boundary regularity for degenerate and singular parabolic equations. Calc. Var. Partial Differ. Equ. 52, 797-827 (2015)

11. Buörn, A., Björn, J., Parviainen, M.: The tusk condition and Petrovskiŭ criterion for the normalized $p$-parabolic equation. Preprint, (2017). arXiv:1712.06807

12. Bögelein, V., DuZaAr, F., Gianazza, U.: Porous medium type equations with measure data and potential estimates. SIAM J. Math. Anal. 45, 3283-3330 (2013)

13. Bögelein, V., Duzaar, F., Gianazza, U.: Very weak solutions of singular porous medium equations with measure data. Commun. Pure Appl. Anal. 14, 23-49 (2015)

14. Bögelein, V., Lukkari, T., Scheven, C.: The obstacle problem for the porous medium equation. Math. Ann. 363, 455-499 (2015)

15. Bögelein, V., Lehtelë, P., Sturm, S.: Regularity of weak solutions and supersolutions to the porous medium equation. Preprint, (2018). arXiv:1801.05249

16. Dahlberg, B.E.J., Kenig, C.E.: Non-negative solutions of the porous medium equation. Commun. Partial Differ. Equ. 9, 409-437 (1984)

17. Dahlberg, B.E.J., Kenig, C.E.: Non-negative solutions of the initial-Dirichlet problem for generalized porous medium equations in cylinders. Rev. Mat. Iberoam. 2, 267-305 (1986)

18. Dahlberg, B.E.J., Kenig, C.E.: Non-negative solutions to fast diffusions. Rev. Mat. Iberoam. 4, 11-29 (1988)

19. Daskalopoulos, P., Kenig, C.E.: Degenerate Diffusions. EMS Tracts in Mathematics 1, European Math. Soc., Zürich, 2007

20. DiBenedetto, E.: A boundary modulus of continuity for a class of singular parabolic equations. J. Differ. Equ. 63, 418-447 (1986)

21. DiBenedetto, E.: Degenerate Parabolic Equations. Universitext, Springer, New York, 1993

22. DiBenedetto, E.: Real Analysis. Birkhäuser Advanced Texts: Basler Lehrbücher, 2nd edn., Birkhäuser/Springer, New York, 2016

23. DiBenedetto, E., Gianazza, U., Vespri, V.: Harnack's Inequality for Degenerate and Singular Parabolic Equations. Springer Monographs in Mathematics, Springer, New York, 2012

24. Gariepy, R., Ziemer, W.P.: A regularity condition at the boundary for solutions of quasilinear elliptic equations. Arch. Ration. Mech. Anal. 67, 25-39 (1977)

25. Gilbarg, D., Trudinger, N.S.: Elliptic Partial Differential Equations of Second Order. 3rd edn., Springer, 1998 
26. Gilding, B.H., Peletier, L.A.: Continuity of solutions of the porous media equation. Ann. Sc. Norm. Super. Pisa Cl. Sci. 8, 659-675 (1981)

27. Heinonen, J., KilPeläinen, T., Martio, O.: Nonlinear Potential Theory of Degenerate Elliptic Equations. 2nd edn., Dover, Mineola, NY, 2006

28. KilpeläInen, T., LindQvist, P.: On the Dirichlet boundary value problem for a degenerate parabolic equation. SIAM J. Math. Anal. 27, 661-683 (1996)

29. Kinnunen, J., LindQvist, P.: Definition and properties of supersolutions to the porous medium equation. J. Reine Angew. Math. 618, 135-168 (2008)

30. Kinnunen, J., Lindevist, P., LukKari, T.: Perron's method for the porous medium equation. J. Eur. Math. Soc. (JEMS) 18, 2953-2969 (2016)

31. Korte, R., Kuusi, T., Parviainen, M.: A connection between a general class of superparabolic functions and supersolutions. J. Evol. Equ. 10, 1-20 (2010)

32. KuUsi, T.: Lower semicontinuity of weak supersolutions to nonlinear parabolic equations. Differ. Integr. Equ. 22, 1211-1222 (2009)

33. Lebesgue, H.: Sur le problème de Dirichlet. C. R. Acad. Sci. Paris 154, 335-337 (1912)

34. Lebesgue, H.: Conditions de régularité, conditions d'irrégularité, conditions d'impossibilité dans le problème de Dirichlet. C. R. Acad. Sci. Paris 178, 349-354 (1924)

35. Lindevist, P.: A criterion of Petrowsky's kind for a degenerate quasilinear parabolic equation. Revista Mat. Iberoam. 11, 569-578 (1995)

36. Littman, W., Stampacchia, G., Weinberger, H.F.: Regular points for elliptic equations with discontinuous coefficients. Ann. Sc. Norm. Super. Pisa Cl. Sci. 17, 43-77 (1963)

37. Malý, J., Ziemer, W.P.: Fine Regularity of Solutions of Elliptic Partial Differential Equations. Math. Surveys and Monographs 51, Amer. Math. Soc., Providence, RI, 1997

38. Petrowsky, I.: Über die Lösungen der ersten Randwertaufgabe der Wärmeleitungsgleichung. Uchebn. Zapiski Moskov. Gos. Univ. 2, 55-59 (1934)

39. Petrowsky, I.: Zur ersten Randwertaufgabe der Wärmeleitungsgleichung. Compos. Math. 1, 383-419 (1935)

40. Poincaré, H.: Sur les équations aux dérivées partielles de la physique mathématique. Amer. J. Math. 12, 211-294 (1890)

41. VÁzQuez, J.L.: The Porous Medium Equation. Oxford Mathematical Monographs, Oxford Univ. Press, Oxford, 2007

42. VESPRI, V.: On the local behaviour of solutions of a certain class of doubly nonlinear parabolic equations. Manuscripta Math. 75, 65-80 (1992)

43. Watson, N.A.: Introduction to Heat Potential Theory. Mathematical Surveys and Monographs 182, Amer. Math. Soc., Providence, RI, 2012

44. Wiener, N.: The Dirichlet problem. J. Math. Phys. 3, 127-146 (1924)

45. Ziemer, W.P.: Interior and boundary continuity of weak solutions of degenerate parabolic equations. Trans. Am. Math. Soc. 271, 733-748 (1982) 
ANDERS BJÖRN and JANA BJÖRN

Department of Mathematics,

Linköping University,

58183 Linköping,

Sweden.

e-mail: anders.bjorn@liu.se; jana.bjorn@liu.se

and

Ugo Gianazza

Department of Mathematics "F. Casorati",

Università di Pavia,

via Ferrata 1,

27100 Pavia,

Italy.

e-mail: gianazza@imati.cnr.it

and

Juhana Siljander

Department of Mathematics and Statistics, University of Jyväskylä,

P.O. Box 35,

40014 Jyväskylä,

Finland.

e-mail: juhana.siljander@jyu.fi

(Received January 25, 2018 / Accepted April 12, 2018)

Published online May 21, 2018

(c) The Author(s) (2018) 